\title{
IMPACTO INSTANTÁNEO Y ACCIÓN CONTINUA EN LA MECÁNICA DE NEWTON ${ }^{1}$
}

\author{
Manuel A. SELLÉS GARCIA \\ Dpto. de Lógica. UNED
}

La presencia de dos definiciones de fuerza dimensionalmente distintas en el Libro I de los Principia de Isaac Newton ha venido dando bastantes preocupaciones a los historiadores. La Ley II establece que:

"El cambio de movimiento es proporcional a la fuerza motriz impresa y ocurre según la línea recta a lo largo de la cual aquella fuerza se imprime» ${ }^{2}$.

Algebraicamente, y en términos más actuales, este concepto de fuerza se ha expresado como:

$$
F=\Delta(m v)
$$

dado que, en este contexto, por "movimiento" hay que entender nuestra actual cantidad de movimiento de un cuerpo, el producto de su masa inercial por su velocidad.

Pero en la Definición VIII, que antecede a esta Ley, Newton escribe que:

"La cantidad motriz de la fuerza centrípeta es la medida de la misma proporcional al movimiento que genera en un tiempo dado" ${ }^{3}$.

\footnotetext{
1 Agradezco a C. Solfs, E. RADA y J. Romo su atenta lectura del manuscrito y sus útiles sugerencias.

2 *Mutationem motus proportionolem esse vi motrici impressae, et fieri secundum lineam rectam qua vis illa imprimatur" NEWTON (1726; 1972), pág. 54. He citado por la traducción de E. Rada, pág. 136.

3 "Vis centripetae quantitas motrix est ipsius mensura proportionalis motui, quem dato tempore generatw. NEWTON (1726; 1972), pág. 44 y, en la traducción de E. RADA, pág. 125.
} 
Lo que se puede representar como:

$$
F=\frac{\Delta(m v)}{\Delta t}
$$

La Ley II establece que la fuerza es proporcional al cambio en la cantidad de movimiento, apuntando a un tratamiento - como se verá- acorde con los fenómenos de impacto. Mientras que la Def. VIII establece que la fuerza es proporcional a la tasa de este cambio, es decir, a la variación de la cantidad de movimiento con el transcurso del tiempo; un concepto en principio más acorde con el tratamiento de fuerzas que, como la centrípeta, actúan sin intermisión. Se ve que la diferencia estriba en la dimensión temporal, que aparece en la segunda y está ausente en la primera.

Salvando el lapso de tres siglos, la fuerza entendida en el sentido de la Def. VIII coincide con nuestro concepto actual; pero no así la que aparece en la Ley II. Dando por sentado que Newton no cometió un error impremeditado - de hecho revisó varias veces esta parte de los Principia - el problema que han debido afrontar los investigadores ha sido el de compatibilizar ambas definiciones de fuerza.

La situación se complica por la presencia en los Principia de dos aproximaciones a la actuación de las fuerzas centrípetas. En una de ellas - se ha denominado la "Construcción por Impulsos Instantáneos (IIC)" - su actuación continua resulta del paso al límite de una serie de impulsos instantáneos impresos a intervalos iguales de tiempo ${ }^{4}$. En la otra, se considera que en intervalos infinitesimales de tiempo la fuerza es constante en magnitud y dirección, aproximándose en tales intervalos la trayectoria por una parábola, en virtud de la ley de Galileo de composición de un movimiento uniforme con otro uniformemente acelerado en línea recta, en el cual los espacios recorridos son como el cuadrado de los tiempos en los que son descritos.

Me he permitido sustituir, en esta traducción, el término "magnitud" por el de "cantidad" por mor, como se verá, de la coherencia con otras partes de este trabajo.

Hay otras dos definiciones relativas a la fuerza centrípeta que preceden a esta. En la Def. VI se establece su «magnitud absolutan, relativa a la eficacia de la causa que la origina; y en la Def. VII se dice que su "magnitud acelerativa" es "su medida proporcional a la velocidad que genera en un tiempo dado.» (Trad. de E. RADA, pág. 125). Dado que por "movimiento" hay que entender la actual "cantidad de movimienton, la magnitud acelerativa es la magnitud motriz por unidad de masa.

4 La denominación se acura en ERLICHSON (1992), si bien la primacía de tal aproximación se defiende en sus trabajos previos, publicados el año anterior. 
Por otra parte, y como es sabido, Newton - junto con Leibniz- recibe el crédito de la autoría del análisis infinitesimal. Los historiadores de la matemática que han analizado su obra coinciden, en general, en considerar su método fluxional equivalente al cálculo diferencial de Leibniz; aunque lo consideran inferior al de éste al hacer del flujo del tiempo la variable independiente de los cambios. Por otra parte, señalan que su procedimiento denominado «de las primeras y últimas razones" constituye un paso en la buena dirección, la de la teoría de límites, que vendría - ya en el siglo XIX- a fundamentar el cálculo sobre una base firme que las diferenciales leibnizianas no le supieron proporcionar. Sin embargo, consideran tal método contaminado por la presencia más o menos oculta de entidades infinitesimales, pese al rechazo explícito de Newton a las mismas.

En un trabajo anterior traté de resolver esta contradicción ${ }^{5}$. Una exploración de la evolución del pensamiento de Newton muestra que sostuvo no una, sino dos concepciones distintas y sucesivas de lo que denominó "momento", si bien sólo calificó de infinitesimal al primero de estos momentos, que fue al que efectivamente renunció. Basándome en las conclusiones de ese trabajo, en las páginas que siguen exploro sus consecuencias dentro del marco de la mecánica de Newton. El objetivo final no es tan sólo el de mostrar la incidencia del rechazo del infinitesimal en los manuscritos mecánicos - los cuales, por otra parte apuntan una datación concreta: 1684, el segundo "annus mirabilis»sino asimismo el de ilustrar el modo en que matemáticas y mecánica se concatenaron para forzar tal transición en el tratamiento de las fuerzas centrípetas, y con ello las vinculaciones entre la aproximación por impulsos a tales fuerzas y su tratamiento como fuerzas continuas a la manera de Galileo.

Antes, sin embargo, conviene recoger - con cierta rapidez-, las consideraciones relativas al rechazo de Newton de esta concepción de infinitesimal.

\section{Newton y el infinitesimal}

Newton formuló su cálculo fluxional en dos versiones distintas que suponen dos estapas sucesivas. A la primera se le puede calificar de cálculo por los

5 SELLES (1999), en prensa. En buena medida, las razones que me llevaron a interesarme por el tema se encuentran en SELLes (1996). Hoy, en todo caso, matizaría algunas de las afirmaciones que alli expresé. 
momentos infinitesimales; se perfiló entre 1665 y 1671 . La segunda sería el cálculo por las razones primeras y últimas, introducido en 1684 en la primera revisión de su manuscrito de Motu, y publicado tres años después en los Principia.

Como es sabido, en el esquema fluxional las magnitudes variables -fluentes, las llamó - cambian con el transcurso del tiempo, siendo su fluxión la velocidad de este cambio. Su primera sistematización apareció en un manuscrito datado en noviembre de $1665^{6}$. Allí planteó lo que hoy denominamos la derivación de una función como la resolución del siguiente problema: Dada una ecuación que expresa la relación entre dos o más líneas $x, y$ descritas en el mismo tiempo por dos o más cuerpos móviles $A, B$, hallar la relación de sus velocidades $p$, q. La solución se basa en que, dado el tiempo, los espacios recorridos son como las velocidades que tienen los cuerpos mientras los recorren, aún cuando el movimiento de éstos no sea uniforme. En tal caso, los espacios que son como las velocidades serán las líneas infinitamente pequenas descritas en lo que denomina cada "momento" de tiempo. De modo que si en un instante dado las líneas descritas son $x$ e $y$, y en un momento el cuerpo $A$, con la velocidad $P$, describe la línea infinitesimal $o$, en el momento siguiente, las líneas descritas serán $(x+o)$ y $(y+o q / p)$, pues se cumple que:

$$
p: q:: o: \frac{o q}{p}
$$

Sentado esto, el procedimiento consiste en sustituir en la ecuación inicial $x$ por $(x+o), y$ por $(y+o g / p)$, simplificar, dividir todos los términos por 0 , y finalmente anular los términos que contengan $o$, por la razón de que son infinitamente pequeños.

En un manuscrito datado en octubre de 1666, el símbolo o pasó a representar el "momento" infinitesimal de tiempo; y la razón entre las velocidades se siguió igualando a la razón entre los espacios recorridos en esta "unidad" infinitesimal de tiempo, es decir ${ }^{7}$ :

$$
p: q:: p o: q o
$$

Finalmente, en la exposición de su nuevo cálculo redactada en 1671, Newton adoptó como unidad el mismo flujo del tiempo ${ }^{8}$. Este flujo sería

6 "To find $y^{e}$ velocitys of bodys by $y^{e}$ lines they describe,n en WHITESIDE (1967-81), Vol.1, págs. 382-389. La primera aparición del método, en las págs. 343-344.

7 Ibid. págs. 400-418; en pág. 414.

8 Conocida como "Tractatus de methodis serierum et fluxionum", si bien Newton no le dio título. En WHITESIDE (1967-81), Vol.3, págs. 32-328. 
exhibido por el movimiento uniforme de una variable escogida como independiente $^{9}$.

Estos cambios respondían al deseo de Newton de enraizar su método en la naturaleza. Su cálculo fluxional buscaba describir la forma en que las magnitudes físicas varían en el mundo fenoménico, estrechando - hasta la identidad- los vínculos entre matemáticas y mecánica. En los Principia manifestó que la geometría se funda en la práctica mecánica, formando parte de una Mecánica universal, entendida como disciplina exacta. Aceptada la génesis de las figuras matemáticas por el movimiento de sus indivisibles ${ }^{10}$, el tiempo pasaba a ocupar en geometría un lugar central como regulador del cambio, como dispensador de "momentos" que, todos iguales por su propia naturaleza, proveían de la unidad de medida básica de todos los cambios.

Ahora bien, esta concepción de "momento" como una entidad infinitesimal no está exenta de problemas. A menos que se acepte la existencia de átomos de tiempo extensos y a la vez indivisibles - posibilidad que Newton se vio obligado a rechazar en sus tempranas anotaciones del llamado "Cuaderno del Trinity"—, este "momento" unidad no podrá ser sino convencional ${ }^{11}$.

Aún entendiéndose divisibles, la admisión de los infinitesimales llevó a considerar a las figuras geométricas como compuestas de estos elementos. Así, por ejemplo, las curvas se identificaban con líneas poligonales formadas por infinitos segmentos rectilíneos infinitamente pequeños. Dichos segmentos, sin embargo, estaban por encima del "nivel dimensional» de los puntos - tenían dimensiones de línea - y, desde nuestro punto de vista actual, habida cuenta del isomorfismo entre el conjunto de los números reales y los puntos de una recta, no serían sino cantidades muy pequeñas, pero a las postre finitas ${ }^{12}$. En

9 Adopción que implica una circularidad. Véase SELLEs (1999).

10 Respecto de la línea, el punto es un indivisible, y aquélla se puede concebir como la traza de un punto en movimiento. Análogamente sucede con la línea respecto de la superficie y con la superficie respecto del sólido.

11 Se trata de su primer cuaderno de notas, en donde registró sus lecturas y reflexiones. $\mathrm{Ha}$ sido editado, con un amplio estudio introductorio, por MCGUIRE y TAMNY (1983). Para una interpretación alternativa a la allí presentada de algunos puntos en relación con este tema, véase SELLES (1999).

12 De hecho, tal representación explica en parte los problemas de fundamentación del cálculo -desde la perspectiva geométrica- de los siglos XVII y XVIII. Como es sabido, tales problemas sólo se resolvieron desechando los infinitesimales en favor de una teoría basada en el concepto de límite. Los infinitesimales, bajo la forma de las diferenciales leibnizianas, siguieron 
una primera etapa, Newton consideró a estas líneas poligonales como las "verdaderas" curvas a tono con muchos de sus contemporáneos. Pero luego pasó a considerarlas como aproximaciones, en las que la curvatura variaría a saltos, fraccionadamente, mientras que en las curvas rigurosas lo haría continuamente, punto a punto. Con tal concepción geométrica del infinitesimal, homogéneo con la magnitud que lo genera mediante una división infinita, Newton llegó a considerar que, por ejemplo, la sustitución de un elemento infinitesimal de arco por su cuerda o su tangente suponía un cierto error al que juzgó inadmisible en una disciplina exacta como la geometría. Tal insatisfacción le llevó a transformar su método fluxional en el método de las razones primeras y últimas, basado en una teorfa de límites.

En relación con el método fluxional, la diferencia entre ambos procedimientos se puede ejemplificar con el caso de la velocidad instantánea. Si escribimos $\nu=d x / d t$, entonces en la interpretación, digamos, "infinitesimal", consideramos a la trayectoria descrita por el móvil como una línea poligonal en la cual el tramo infinitesimal $d x$ correspondiente al "momento" infinitesimal $d t$ se recorrerá efectivamente con velocidad constante $v$. Será una "verdadera" velocidad, en la medida en que $d t$ sea un verdadero intervalo de tiempo. Este resulta de la división, llevada al infinito, de un intervalo finito de tiempo determinado. Nótese que es un proceso de división infinito que de alguna manera no se ha completado pues, por ejemplo, todavía tiene sentido dividir $d t$ por dos. A este tipo de infinito (digamos que es un número mayor que cualquier número propuesto) convendremos en denominarlo potencial. Pero ahora supongamos que el susodicho intervalo finito de tiempo se sigue dividiendo - puesto que nuestro $d t$ todavía es divisible - hasta el límite en que ya no es posible una división ulterior. Convendremos en llamar al infinito involucrado en tal división un infinito actual (un número mayor que todo número). El resultado final de tal división serfa indivisible: no ya un intervalo infinitesimal, sino un instante, un punto. Con esto se ha trascendido el nivel de la curva entendida $\longrightarrow$ aproximada - como una línea poligonal para llegar a la curva

presentes sin embargo en la física - y aún en cierta medida en las matemáticas- de manera injustificada hasta que, en la década de los 1960 de nuestro siglo, A. Robinson mostró que su existencia podía fundarse en bases lógicamente firmes. Pero ello implicaría incluirlos dentro de los puntos -dotándolos así de estructura - o, lo que resulta equivalente, incluir nuevos puntos en la inmediata vecindad de cada punto de una recta real de por sí ya densa. Tal posibilidad no se contemplaba en la época que estamos tratando. En relación con Newton, desarrollo una argumentación más detenida sobre esta cuestión en SELLÉs (1999). 
«verdadera» o rigurosa, aquélla cuya curvatura — puntos singulares apartecambia continuamente, y en la que el término "continuidad", por contraposición con la anterior imagen poligonal, cobra un nuevo sentido. En tales circunstancias, los "momentos» se han convertido en instantes, dándose ahora la circunstancia de que en un instante no se puede hablar de velocidad; pues, al no tener extensión, en él no se recorre ningún espacio. La manera de atribuirle una velocidad es adjudicarle la que hubiese adoptado si, libre de ligaduras, el móvil que recorre la curva hubiese seguido con velocidad uniforme - movimiento inercial - la tangente a la trayectoria en el punto correspondiente a ese instante. Velocidad medida por el espacio que hubiese recorrido según esta tangente en un intervalo de tiempo dado. Pero velocidad, al cabo, que no deja de ser una virtualidad, una potencialidad, una capacidad o, para usar los términos de la época, un "power", un «endeavour» o un "conatus».

Como se ha mencionado, en su primera versión del cálculo fluxional Newton estimó la razón entre las fluxiones a través de la razón entre los momentos de sus fluentes, entendidos dichos momentos como las cantidades infinitesimales en que varían durante un intervalo de tiempo infinitesimal. Pero si la línea poligonal infinitangular era sólo una aproximación a la verdadera curva, a la curva rigurosa, entonces la razón entre los momentos sólo sería aproximadamente igual a la razón entre las fluentes. Buscando una proporción rigurosa, exacta, en algún momento anterior a los Principia Newton introdujo, en uno de sus manuscritos, una nueva concepción de los momentos:

"Los momentos de cantidades son sus principios de generación o alteración en un flujo continuo: como el tiempo presente lo es del pasado y el futuro, el movimiento presente del pasado y del futuro, la fuerza centrípeta o cualquier otra fuerza momentánea del impetus, el punto de la línea, la línea de la superficie, la superficie del sólido y el ángulo de contacto del ángulo rectilíneon ${ }^{13}$.

13 «Momenta quantitatum sunt ipsarum principia generantia vel alternantia fluxu continuo: ut tempus praesens praeteriti et futuri, motus praesens praeteriti et futuri, vis centripeta aut alia quaevis momentanea impetus, punctum lineae, linea superficiei, superficies solidi et angulus contactus anguli rectilinei». El ángulo de contacto era el que se suponía formado entre la circunferencia y su tangente en un punto, que era su vértice. Este texto de Newton se encuentra en una revisión del manuscrito "de Motu corporum", y fue cancelado. Pero el concepto es el mismo que se expresa en el Lema II del Libro II de los Principia. En HerIvel (1965), pág. 306. 
Con esta nueva concepción de momento, la proporción de las razones entre fluxiones por un lado y momentos por el otro se torna estricta, sin ningún tipo de error o aproximación introducido por los infinitesimales. Pues ahora los momentos pierden esta condición para tornarse indivisibles, inhomogéneos con la magnitud de la que proceden, pero capaces de generarla gracias y a traves del mecanismo potenciador y regulador del flujo del tiempo. De generarla en cantidades finitas:

"Las partículas no son momentos, sino las cantidades mismas generadas por los momentos. Han de entenderse como los mismos principios nacientes de las magnitudes finitas ${ }^{14}$.

La cuestión es ahora cómo determinar la razón entre unos momentos que son indivisibles, sin magnitud respecto de las cantidades finitas que, gracias al transcurrir del tiempo, generan. Newton encontró la solución en la aplicación de un concepto de límite. La razón entre las cantidades finitas generadas por los momentos en un intervalo de tiempo dado será sólo aproximadamente igual a la razón entre los momentos, pero tanto más aproximada cuanto menor sea dicho intervalo de tiempo. En el límite, cuando estas cantidades "nacen" o "se desvanecen", la igualdad será estricta. Éste es el método de las razones primeras y últimas.

De acuerdo con esto, deberían poderse distinguir dos etapas en las investigaciones dinámicas de Newton, correspondientes a estas dos versiones -con y sin sus infinitesimales- del cálculo. Esta circunstancia no ha sido considerada por los historiadores desde este punto de vista, por lo que cabe esperar que su obra anterior a los Principia pueda interpretarse con alguna nueva matización que arroje luz sobre las dificultades ya mencionadas en la introducción, halladas en los Principia mismos.

\section{El impacto y la fuerza}

En el contexto mecanicista de la época, tanto plenistas como vacuistas conceblan a la materia inerte, desprovista de toda actividad; por consiguiente, sus

14 Particulae finitae non sunt momenta, sed quantitates ipsae ex momentis genitae. Intelligenda sunt principia jamjam nascentia finitarum magnitudinumw. NEWTON (1726; 1972), pág. 365; trad. de E. RADA, pág. 431. 
interacciones se explicaban a través del contacto, es decir, por medio de la presión o del impacto. Por ello, y también por los errores contenidos en las siete reglas del choque que Descartes publicó en sus Principios de Filosofla, el estudio del impacto despertó un vivo interés ${ }^{15}$. Newton se interesó tempranamente por estas cuestiones, ocupándose bastante del tratamiento del choque en sus primeros manuscritos sobre mecánica ${ }^{16}$. En ellos, como es sabido, corrigió el error de Descartes en la aplicación de la ley de conservación del movimiento, haciendo intervenir la suma algebraica de los movimientos habida cuenta de su dirección y sentido o, como se la denominaba entonces, "determinación". Recuérdese que Newton entendía por "movimiento" la actual cantidad de movimiento, el producto de la masa del cuerpo por la velocidad; si bien en esta primera etapa el concepto de masa todavía no estaba del todo bien perfilado.

En los estudios del impacto, el procedimiento consistía en determinar la diferencia entre los movimientos de los dos cuerpos en instantes indeterminados antes y después del choque. La consideración de lo sucedido en el choque mismo era como mucho secundaria, y sólo intervenía a través de la naturaleza de los cuerpos implicados. Descartes formulo sus leyes del choque para cuerpos

15 Para un panorama general, véase el Cap. I de ScoTT (1970).

16 En HerIVEl (1965), se dividen los manuscritos dinámicos de Newton en dos grupos. El primero de ellos comprendería los redactados antes de la carta a Huygens en 1673; considerándolos, en su mayoría, muy anteriores a esta fecha. El segundo grupo comprendería los escritos entre mayo de 1684 y el verano de 1685 . Herivel los numera sucesivamente del I al XI, analizando su datación probable en el Cap. 6. Los correspondientes al primer grupo son los siguientes:

I. Anotaciones sobre mecánica en Add.3996, denominado el "Cuaderno del Trinity". Datan de 1664 o, como tarde, de principios de 1665. El manuscrito ha sido publicado en su totalidad por MCGUIRE y TAMNY (1983).

Il. Manuscritos dinámicos en el "Waste Book". Se dividen en siete partes (Ila-Ilg), cubriendo los folios 1-38. En IIb aparece la fecha del 20 de Enero de 1664 [O.S.], y considera posible que representen versiones de borradores anteriores compuestos a fines de 1664 o en enero de 1665 .

III. Ms. Add. 3958, fo 45, denominado "Vellum manuscript". Compuesto probablemente en 1665 o 1666.

IV. En dos partes, en la sección 5 de Ms. Add. 3958. Anteriores a 1669.

V. El *The Laws of Motion", Ms. Add.3958 (5), ff. 81-83.

VI. Conocido como el "De gravitatione et aequipondio fluidorum», Ms. Add. 4003. Herivel lo data entre 1665 y 1669, si bien WeSTFAll (1980), pág. 301 lo sitúa, como pronto, en 1668. Publicado integramente en Hall y Hall (1962).

En lo sucesivo se citarán por la paginación de la edición de Herivel, antecedida por el número correspondiente al manuscrito. 
perfectamente duros. Por tales cuerpos entendía aquellos cuyas partes se encuentran en reposo; si a dichas partes se les comunica movimiento y se agitan, entonces se convierten en fluidos. En todo caso, los cuerpos son siempre divisibles. En el choque entre cuerpos duros, estos rebotan y el movimiento se conserva - lo que hoy llamamos choques perfectamente elásticos-- pero el rebote no se produce porque éstos se compriman. Tal concepción convierte a los choques en instantáneos, con la secuela de que los cambios en los movimientos son discontinuos. Esto a Descartes no le suponía ningún problema, pues creía que Dios recreaba el mundo a cada instante, no existiendo así una vinculación dinámica necesaria entre el estado de un cuerpo en un instante y su estado en el instante siguiente. Así, un cuerpo que parte del reposo no tiene por qué pasar por todos los grados de velocidad, como pretendía Galileo ${ }^{17}$.

Para sus oponentes atomistas, con sus átomos físicamente indivisibles, la dureza tenía otro sentido, asociándose a la solidez y a la indeformabilidad -ausencia de poros-y por consiguiente a una perfecta resistencia a la fractura. Pero en una primera etapa compartirán con Descartes la convicción de que en los choques entre cuerpos duros no hay pérdida alguna de movimiento ${ }^{18}$.

En una de las entradas del "Waste Book", Newton consideró un choque frontal entre dos cuerpos iguales, de forma esférica, elásticos y deformables, que se oponen con la misma velocidad. En tal caso, escribe, los cuerpos sufrirán una deformación durante el impacto, tornándose esferoidales:

“(...) encontrándose uno con otro cederán y serán comprimidos adoptando una figura esferoidal, y en este momento del período del confrontamiento de su movimiento uno hacia el otro su figura será la más esferoidal, y su presión uno sobre otro será máxima (...)" ${ }^{19}$.

17 Véase SHEA (1993), págs. 354-55 sobre el concepto de dureza, y págs 412-420 sobre sus leyes del choque y la discontinuidad de los cambios en el movimiento.

18 Tales son las características de los átomos que, para Newton, componen toda materia, si bien en la Optica considerará su colisión, al contrario que en estos primeros manuscritos, perfectamente inelástica. Su teoría de la materia la expone en la Cuestión 23 de la 1. a edición, que luego pasará a ser la 31. La pérdida del movimiento en los choques - salvo en el caso de cuerpos perfectamente elásticos- se encuentra en NEWTON (1730; 1977), pág. 343. Sobre la dureza de los átomos, véanse las págs. 345-6.

19 a(...) meeting one another they will relent and be pressed into a sphaeroidicall figure, and in that moment in which there is a period put to their motion towards one another their figure will be most sphaeroidical and theire pression one upon the other is at greatest (...)". Herrvel (1965), IId, pág. 142. 
El impacto de estos cuerpos requiere, pues, un cierto intervalo de tiempo. Pero si se suponen indeformables, absolutamente sólidos, entonces su contacto se efectuará en un único punto y su encuentro será instantáneo:

«(...) supuesto que sean esféricos y absolutamente sólidos, entonces en el periodo de mvimiento uno hacia el otro (esto es, en el momento de su encuentro) su presión está en el máximo o mejor dicho esto se produce con la fuerza total por la cual su movimiento es detenido (pues su movimiento total fue detenido por la fuerza de su presión uno sobre otro en este momento, y no pueden darse diversos grados de presión entre dos cuerpos en un momento)» ${ }^{20}$.

Obsérvese que la diferencia entre ambos tipos de choque - con y sin deformación - consiste en que la fuerza necesaria para detener los cuerpos, cuya aplicación en el caso de los deformables requiere un cierto tiempo, se aplica instantáneamente cuando son duros. A igualdad de masas y velocidades iniciales, el efecto final de estas dos fuerzas es el mismo ${ }^{21}$. Si las fuerzas, entendidas como causas, son proporcionales a y se miden por sus efectos, tal igualdad supondrá la de las fuerzas mismas. Las fuerzas cuya acción se extiende a lo largo de un intervalo de tiempo y aquéllas otras que son instantáneas resultan, pues, comparables. $\mathrm{Y}$ en ambos casos su medida entraña la intervención de un intervalo de tiempo, que no tiene nada que ver con aquel durante el cual actúa la fuerza, sino que se considera una vez que la fuerza ha dejado de actuar y el cuerpo se mueve con movimiento inercial. En la entrada inmediatamente anterior a las anotaciones sobre el impacto que hemos considerado, Newton escribió una serie de definiciones. En la segunda de ellas se establece la medida de las velocidades:

"Una Cantidad [interprétese este término como "masa»] es tanto más rápida que otra, como la distancia que recorre es mayor que la distancia que atraviesa la otra en el mismo tiempon ${ }^{22}$.

20 "(...) suppose they be sphaericall and absolutely sollid then at the period of theire motion towards one another (that is at the moment of theire meeting) theire pression is at greatest, or rather 'tis done with the whole force by which theire motion is stopt (for theire whole motion was stoped by the force of theire pressure upon one another in this one moment, and there cannot be succeede divers degrees of pressure twixt two bodys in one moment)". Ibid, págs. 142-3.

21 No asi desde el punto de vista actual. Si la fuerza aplicada sobre un cuerpo es medida por la tasa de cambio de su cantidad de movimiento, entonces una variación instantánea de éste requerirá una fuerza infinita.

22 "One Quantity is soe much swifter than another, as the distance throught which it passeth is greater than the distance through which the other passeth in the same time.» HERIVEL (1965), IIc, pág. 136. 
Y en la tercera la medida de las cantidades de movimiento:

"Una cantidad tiene tanto más movimiento que otra como la distancia a través de la que se mueve compuesta en su cantidad, es a la distancia a través de la cual la otra se mueve en el mismo tiempo, compuesta con su cantidad. (...). O bien el movimiento de una cantidad a otra es como sus capacidades de perseverar en ese estadom 23.

La definición 9 atañe a la fuerza:

"Fuerza es la presión o empuje de un cuerpo sobre otron ${ }^{24}$

La proporcionalidad entre la fuerza y la cantidad de movimiento se desarrolla en las dos entradas siguientes. Newton distingue allí entre la fuerza de un cuerpo, que es proporcional a su movimiento y por tanto a la distancia que recorre en un tiempo dado, y la fuerza ejercida sobre un cuerpo, proporcional al movimiento en él generado o destruído. Establece que el cambio de movimiento se produce en la dirección en que actúa la fuerza, y esboza el principio de acción y reacción ${ }^{25}$.

Otro manuscrito que contiene una serie de definiciones fue redactado c.1669. Se conoce por las palabras con que comienza: «De gravitatione et aequipondio fluidorum». En él la definición de fuerza es mucho más completa:

«Def.5. Fuerza es el principio causal del movimiento y del reposo, y es, o bien principio externo que, impreso en un cierto cuerpo, genera o destruye su movimiento, o al menos lo cambia en algo; o es el principio interno por el cual el movimiento o reposo impreso en el cuerpo es conservado, y por el cual cada entidad tiende a perseverar en su estado actual, y resiste a cualquier obstáculo» ${ }^{26}$.

23 «One quantity hath so much more motion than another, as the distance through which it moveth drawne into its quantity, is to the distance through witch the other moveth in the same time, drawne into its quantity. (...). Or the motion of one quantity to another is as their powers to persever in that state.» Ibid, págs. 136-137.

24 "Force is the pressure or crouding of one body upon another." Ibid, pág. 138.

25 Véase el comentario introductorio de HERIVE (1965) a la entrada Ile, en las págs. 152-153.

26 «Def.5. Vis est motus et quietis causale principium. Estque vel externum quod in aliquod corpus impressum motum ejus vel generat vel destruit, vel aliquo saltem modo mutat; vel est internum principium quo motus vel quies corpori insita conservatur, et quodlibet ens in suo 
En la definición 8 denomina «inercia» a esta fuerza que mantiene el estado del cuerpo.

El conatus es un caso particular de fuerza:

"Def.6. Conatus es una fuerza impedida, o una fuerza en tanto en cuanto es resistidan ${ }^{27}$.

y la presión es un tipo de conatus.

"Def. 9. Presión es el conatus de partes adyacentes que tienden a penetrar mutuamente sus espacios. Pues si fuesen capaces de penetrarse cesaría la presion" ${ }^{28}$.

Finalmente, la gravedad es una fuerza intrínseca que inclina a los cuerpos a descender.

Las cantidades del movimiento, la fuerza, el conatus, el impetus, la inercia, la presión y la gravedad, definidas anteriormente, se pueden determinar de dos maneras: atendiendo a su intensidad, o a su extensión ${ }^{29}$. Según la definición 11, la intensidad es "el grado de su cualidad» y la extensión es «la cantidad de espacio o tiempo en la cual actúa" ${ }^{30}$. A continuación establece que la "cantidad absoluta» es la que se compone (es el producto) de la intensidad y la extensión. De este modo - prosigue- el movimiento es más intenso o remiso según se atraviese un mayor o menor espacio en el mismo tiempo; y es más o menos extenso en la medida en que el cuerpo que se mueve es mayor o menor. Pero además -escribe un poco más adelante- es posible que en ocasiones haya que calcular estas capacidades respecto de la duración de intervalos; en

statu perseverare conatur et inpeditum reluctatur.w HERIVEL (1965), VI, pág. 224; y HaLL y HALI (1962), pág. 114.

27 "Def. 6. Conatus est vis impedita sive vis quatenus resistitur." Ibid. La influencia cartesiana en la formulación del concepto es clara.

28 «Def. 9. Pressio est partium contiguarum conatus ad ipsarum dimensionies mutuo penetrandum. Nam si possent penetrare cessaret pressio.» Ibid.

29 Newton define al impetus como "fuerza en la medida en que es impresa sobre algo" [Impetus est vis quatenus in aliud imprimatur] y la inercia como la kfuerza interna de un cuerpo que se opone a que su estado no sea cambiado fácilmente por cualquier fuerza externa" [Inertia est vis interna corporis ne status ejus externa vi illata facile mutetur]. Ibid.

30 «Intensio potestatis alicujus praedictae est ejus qualitatis gradus», y «Extensio ejus est spatij vel temporis quantitas in quo exercetur.» Ibid, resp. págs. 225 y 115. 
cuyo caso habrá que componer la intensidad, la extensión y la duración. El ejemplo que da se refiere al movimiento:

"Por ejemplo si un cuerpo 2 con velocidad 3 se mueve durante el tiempo 4, el movimiento total será $2 \times 3 \times 4$, o 12 [sic]" ${ }^{31}$.

A continuación establece que la velocidad «es la intensidad del movimientom ${ }^{32}$.

Sin duda el lector ha reconocido aquí elementos de la antigua doctrina medieval de la configuración de las formas, encaminada a caracterizar los cambios de las cualidades en los cuerpos ${ }^{33}$. En ella se consideraba que una cualidad tenía dos dimensiones: longitud, de acuerdo con la extensión del sujeto de la cualidad, y latitud, según el grado de intensidad. La cantidad de una cualidad - "cantidad absoluta", la denomina Newton- estaba representada por la figura bidimensional formada por la extensión y los distintos grados de intensidad en los distintos puntos de esta extensión. Así, la "cantidad total" de una cualidad extendida sobre un cuerpo y que actuase a lo largo de una cierta duración vendría representada por un sólido. En el ejemplo anterior, la intensidad del movimiento es la velocidad (3), su cantidad absoluta es su cantidad de movimiento $(2 \times 3)$, y el movimiento total al cabo del tiempo 4 será $(2 \times 3 \times$ 4). Velocidad, movimiento y movimiento total tienen, respectivamente, las dimensiones de la línea, la superficie y el sólido. Nótese, además, que en este caso, aunque la velocidad del cuerpo es constante y por tanto su movimiento uniforme, el movimiento total así definido es una magnitud que crece linealmente con el tiempo ${ }^{34}$.

A la luz de lo anterior, comparemos el impacto entre dos cuerpos elásticos y dos cuerpos duros que comentamos anteriormente. Entre cuerpos duros, la fuerza motriz será la fuerza total, aquí igual a su cantidad absoluta, producto de la extensión (la masa), por la intensidad:

31 "Quemadmodum si corpus 2 velocitate 3 per tempus 4 movetur: totus motus erit $2 \times 3 \times 4$, sive 12." Ibid., resp. págs. 226 y 115.

32 "Velocitas est motus intesio, ac tarditas remissio ejus." Ibid.

33 HeRIVEL (1965), p. 235, nota 32, advierte este uso por parte de Newton.

34 Hall y Hall $(1962,1978)$, pág. 85, comentan que estas definiciones uno presentan por sí mismas un interés especialn y que Newton no aclara si la cantidad absoluta de movimiento debería definirse como mv o mvt. La distinción que se hace aquí entre kcantidad total" y *cantidad absolutan - que coinciden cuando no interviene la duración- aclara la cuestión. 


$$
F_{d}=\Delta(m v)=m \cdot I_{d}
$$

mientras que, entre cuerpos deformables y elásticos, la fuerza total (suponiendo la intensidad constante a lo largo de todo el intervalo de tiempo durante el que actúa la fuerza), resulta:

$$
F_{e}=\Delta(m v)=m \cdot I_{e} \cdot \Delta t
$$

Medidos después del impacto, los cambios en el movimiento son los mismos, por lo que $F_{d}=F_{e}$ y:

$$
I_{\bar{d}}=I_{e} \cdot \Delta t
$$

Se ve que $I_{d}$ es de una dimensión superior a $I_{e}$. Si ésta se representa por una línea, entonces aquélla lo sería por una superficie.

Demos ahora un salto en el tiempo y comparemos estas definiciones con los Principia. El lenguaje ha cambiado algo, pero no mucho. Newton ahora no da una definición general de qué es la fuerza, así que admitiremos provisionalmente la def. 5 del de Gravitatione, que es bastante completa. La fuerza es un "principio causal" que genera, destruye o cambia el movimiento de un cuerpo (nos estamos refiriendo a fuerzas impresas, dejando de lado la fuerza de inercia). Como "principio", es independiente del cuerpo considerado o de las causas de su acción; así, la fuerza es distinta de su cantidad, que es resultado de su medida. Pero como "causa», es proporcional al efecto así que, caeteris paribus, la igualdad o razón de sus efectos supondrá la igualdad o razón de las fuerzas. Newton llama "fuerzas" a las intensidades. En las Definiciones, distingue tres tipos de fuerza: Insita, impresa y centrípeta. La fuerza impresa tiene distintos origenes: tales como un golpe, una presión o la fuerza centrípeta. Así, la fuerza centrípeta origina o es una fuerza impresa, una acción que se ejerce sobre un cuerpo, al igual que un impacto, instantáneo o no. Por "fuerza centrípeta", Newton entiende la intensidad. La Def. VI atañe a su cantidad absoluta, el producto de la intensidad por la extensión; el ejemplo del imán es claro: la cantidad absoluta varía según la intensidad de fuerza magnética o según la masa del imán. Las definiciones VII y VIII se refieren a cantidades totales, y por ello hacen intervenir al tiempo: la cantidad de aceleración que puede producir en un tiempo dado, o respectivamente la cantidad motriz, la cantidad de movimiento que puede originar en un tiempo dado. Éste pudo ser 
el razonamiento de Newton: dado el intervalo de tiempo, la cantidad motriz varía como la [intensidad de la] fuerza, y dada ésta, varía como el tiempo, y por lo tanto cuando ninguno de ellos está dado, la cantidad motriz varía conjuntamente como la [intensidad de la] fuerza y como el tiempo. Así, la ufuerza total" centripeta o, como con mayor precisión denomina Newton en los Principia, la medida de la cantidad motriz de la fuerza centrípeta, $F_{T C}$ resulta:

$$
F_{T C} \propto I_{C} \cdot \Delta t
$$

Nótese que la ec. (8) coincide con la (6), pero supone una lectura de la Def. VIII bien distinta de la tradicional, que se presentó en la ec. (2). Cabe suponer que esto se debe a que hoy en día siempre definimos las fuerzas como magnitudes instantáneas, y por ello se ha interpretado que, en esta Definición, Newton se estaba refiriendo a $I_{c}$ cuando en realidad pensaba cómo medir $F_{T C}$ De acuerdo con los métodos geométricos al uso, Newton determina estas cantidades motrices de la fuerza centrípeta comparándolas unas con otras en el mismo intervalo de tiempo, llegando así a una razón entre intensidades o, como él las llama, "fuerzas" a secas. Pero es que, además, para mayor brevedad, omite el término "cantidad" y así, por ejemplo, en lugar de hablar de la "cantidad morriz de la fuerza" habla de la "fuerza motriz»:

«Es conveniente, para ser breves, llamar a estas cantidades fuerzas motrices, acelerativas y absolutas; (...)" ${ }^{35}$.

Vayamos ahora al enunciado de la Ley II citado al comienzo de la introducción. Se ve que la igualdad (1) es la misma que la primera de las igualdades en (5) y en (6). Además, Newton habla de fuerza motriz impresa. De modo que el enunciado es válido tanto para fuerzas instantáneas $F_{d}$ como para fuerzas continuas $F_{i}$ La fuerza motriz impresa es siempre, para Newton, una "fuerza totalm; por la sencilla razón de que la fuerza centrípeta en un punto -en nuestros términos, la intensidad $I_{c}-$ no tiene virtud motriz. Una fuerza centrípeta sólo moverá un cuerpo si actúa a lo largo de un intervalo de tiempo, no en un instante. De este modo, no hay ninguna contradicción entre la Def. VIII (y su aplicación a lo largo de los Principia) y la Ley II. Pero es que además

35 «Hace virium quantitates brevitatis gratia nominare licet vires motrices, acceleratrices, \& absolutas; (...).» NEWTON (1726; 1972), págs. 44-45. Trad. de E. RADA, págs. 125-126. 
Newton explicita que la Ley se refiere a ambos tipos de fuerzas, instantáneas y continuas, en las líneas que escribe a continuación del enunciado de esta Ley:

"Si una fuerza cualquiera produce un movimiento dado, doblada producirá el doble y triplicada el triple, tanto si se aplica de una sola vez como si se aplica por grados y sucesivamenten ${ }^{36}$.

En 1991, H. Erlichson publicó un trabajo en el que, en relación con el modelo de impactos instantáneos, puso de relieve que la concepción de fuerza que desempena un papel predominante en los Principia de Newton es la cantidad de fuerza motriz de la Def. VIII, una cantidad escalar que crece con el tiempo. Distinguió así entre la fuerza - nuestra intensidad-y su cantidad motriz, presentando una lectura correcta de la Def. VIII. Sefialó que, cuando se comparan dos fuerzas uniformes, éstas son proporcionales a sus cantidades motrices para intervalos de tiempo iguales; por otra parte, la fuerza individual en un punto se obtiene tomando el límite de su cantidad motriz cuando el intervalo de tiempo tiende a cero ${ }^{37}$. De hecho, el interés de Erlichson se centra en la defensa del papel que el modelo de impactos instantáneos desempeńó en la obra de Newton, y así tanto en este caso como en otros trabajos ha permanecido ajeno tanto a los problemas de conciliación de la Def. VIII con la Ley II como a los estudios de la historia del cálculo, los cuales ponen de manifiesto que no cabe atribuir a Newton una teoría de límites - como hace Erlichsonen la etapa correspondiente a sus primeros manuscritos mecánicos.

Como se ha visto, la presente interpretación reconcilia la Definición con la Ley, mostrando que tal reconciliación es acorde con toda la tradición acerca de la medida de magnitudes que se expresan geométricamente, y que tal tradición se halla presente en los manuscritos newtonianos previos al de Motu. Hay un solo concepto de fuerza, la "fuerza total", proporcional al cambio "total" en la cantidad del movimiento, pero todavía, atendiendo a la duración, hay dos tipos de fuerzas, según que actúen o no instantáneamente, cuyas intensidades son inconmensurables. Hoy no aceptamos la existencia de impulsos instantáneos, por lo que nuestra concepción de fuerza responde a la ec. (6) y no a la

36 «Si vis aliqua motum quemvis generet; dupla duplum, tripla triplum generabit, sive simul \& semel, sive gradatim \& sucesive impressa fuerit." Ibich, pág. 54 . Trad. de E. Rada, pág. 136. He sustituido "por grados" el término ugradual" de la traducción para subrayar la continuidad y la vinculación a las teorías medievales de medida de las cualidades. 
(5). Por ello se dice en ocasiones que, si se diese tal impulso, la fuerza sería infinita. Si en la ec. (6) se mantiene constante $F_{e}$ mientras que $\Delta t \rightarrow 0$, entonces $I_{e} \rightarrow \infty$; pero, si se aplica la ec. (5), entonces $I_{d}$ es finita. De hecho, en el límite, cuando $\Delta t=0$, la fuerza total se torna indeterminada: $\infty .0$. Newton no se confundió nunca manejando ambos tipos de fuerza y, a diferencia de lo que sostienen autores como Herivel o Westfall, tenía las ideas bastante claras a este respecto, cuanto menos en la etapa iniciada con la redacción del de Motu en 1684. Pero antes de adentrarse en esta cuestión, conviene plantearse las razones que pudo tener para acoger en su mecánica a los impulsos instantáneos.

Según una interpretación tradicional, Newton introdujo los impulsos instantáneos como la idealización de choques reales, una idea que pudieron sugerir sus estudios sobre el impacto en sus primeros manuscritos mecánicos. El origen de esta concepción puede hallarse en las manifestaciones de Herivel, quien interpretó la Ley II precisamente en este sentido ${ }^{38}$. En realidad hay también otros motivos contextuales que inciden en la introducción por parte de Newton de impulsos instantáneos. Westfall apunta en esta dirección al vincular las dos concepciones de fuerza de Newton a la existencia de dos posibles modelos de conceptualización de la fuerza. Estos modelos derivarlan, el uno del estudio del impacto, el otro del análisis de Galileo del movimiento uniformemente acelerado ${ }^{39}$. Veamos primero el caso del impacto. Si los impulsos instantáneos surgen únicamente como conceptualización de choques reales, ̨̧habría que entender asimismo que los "cuerpos duros" son una idealización de cuerpos o partículas físicas? Si la respuesta es sí, entonces estamos contradiciendo todo el contexto de la época al que ya aludíamos al principio de este apartado: caen por su base tanto el sistema de Descartes como la resistencia a la fractura de los átomos de sus oponentes. En la Optica y otros lugares, Newton habló de los átomos como cuerpos perfectamente duros. Pero, si la dureza era una propiedad esencial de los átomos, no sucedía lo mismo con las particulas que estos formaban por sucesivos órdenes de composición, y que eran las que realmente intervenían en las interacciones. Así, en el Escolio a los axiomas o leyes del movimiento de los Principia, Newton admitió que:

37 ERLiChSON (1991a), pág. 843.

38 uusa el término [fuerza] en el sentido de un impulso que mide el efecto total de una fuerza variable, fisica. Es este último tipo de fuerza motriz d que presenta en la segunda ley del movimiento.» HERIVEL (1965), pág. 140, nota 10. Sobre esta concepción, que comparten autores como Cohen y Erlichson, volveremos más adelante.

39 Así, el primero responderfa a la interpretación clásica de la Ley II, y el segundo a la Definición VIII de los Principia. 
"los cuerpos (...) absolutamente duros o por lo menos perfectamente elásticos, (...) no se hallan en lo más mínimo entre las cosas naturales; (...) ${ }^{40}$.

Por otra parte, en sus manuscritos mecánicos del período 1665-69 se aprecia que, más que una idealización del caso real, la atribución de dureza a los cuerpos cuyo choque estudia es una necesidad; pues, cuando señala en las "Leyes del movimiento", la consideración del choque entre cuerpos fisicos supone un modelo de tremenda complejidad. Tras considerar el impacto de cuerpos duros en rotación, escribe:

"Sólo aquellos cuerpos que son absolutamente duros se reflejan exactamente de acuerdo con estas reglas. Ahora bien, los cuerpos que nos rodean (al constituir un agregado de otros cuerpos menores) tienen una blandura y elasticidad que los hacen ceder, lo que provoca que su contacto sea por algún tiempo y en más de un punto. Y las superficies que se tocan durante el tiempo del contacto deslizan entre sí más o menos o nada en absoluto según su rugosidad. $\mathrm{Y}$ pocos o ninguno de estos cuerpos tienen una elasticidad tan fuerte como para separarlos con el mismo vigor con el que se encontraron. Además de que sus movimientos son estorbados y reducidos continuamente por los medios en que se mueven. Quien quisiese prescribir reglas para la reflexión de estos cuerpos compuestos debe considerar en cuántos puntos se tocan los dos cuerpos al encontrarse, la posición y presión de cada punto con sus planos de contacto, etc.: y cómo todo ello varía en cada momento durante el tiempo de contacto por la mayor o menor blandura y elasticidad de estos cuerpos que les hacen ceder y por sus diversos deslizamientos. $Y$ también qué efecto puede tener el aire u otros medios comprimidos entre los cuerposm ${ }^{41}$.

40 "Porro nequis objiciat regulam, ad quam probandam inventum est hoc experimentum, praesupponere corpora vel absolute dura esse, vel saltem perfecte elastica, cujusmodi nulla reperiuntur in compositionibus naturalibus; (...)m. NEwTON (1726; 1972), pág. 69. Trad. de E. Rada, pág. 149.

41 *Only, these bodys which are absolutely hard are exactly reflected according to these rules. Now the bodys here among us (being an aggregate of smaller other bodyes) have a relenting softnesse and springynesse, which makes their contact be for some time and in more points than one. And the touching surfaces during the time of contact doe slide one upon another more or lesse or not at all according to their roughnesse. And few or none of these bodyes have a springynesse soe strong as to force them one from another with the same vigor that they come together. Besides, that their motions are continually inpeded and slackened by the mediums in which the move. Now he that would prescribe rules for the reflection of these conpound bodies, must consider in how many points the two bodies touch at their meeting, the position and 
De modo que se pueden considerar cambios instantáneos en el movimiento, pero tales cambios sólo se darán, si acaso, en el mundo de la microfísica, el reino de los átomos. Siendo muy escasa la cantidad de materia del universo, y hallándose estos átomos ligados dentro de otras partículas en el nivel, digamos, "molecularn, realmente la colisión entre átomos no debía ser nada frecuente. Esto parece apoyar la idea de que los impulsos instantáneos surgen fundamentalmente de una conceptualización - como se ha visto, más bien de una necesidad- en el estudio de impactos físicos. Sin embargo, este nivel microfísico también nos remite al otro modelo de fuerza presentado por Westfall, el de la caída libre.

Como es sabido, Descartes había explicado la acción de la gravedad por la actuación de impulsos impartidos por partículas muy sutiles, y ya en el "Cuaderno del Trinity" Newton adoptó un modelo de éter material compuesto por partículas cuya acción mecánica, por impulsos, explicaría toda una gama de fenómenos, atracciones magnéticas y eléctricas y la gravedad incluídas. ${ }^{42}$ Resultando así la gravedad del efecto de una infinidad de minúsculos choques o impulsos, esto sugiere su análisis en términos de una acumulación de acciones mínimas, indivisibles o infinitesimales. Así en el caso de la percusión contra el suelo de un cuerpo en caída libre, cuya fuerza -en consonancia con una dinámica surgida como derivación de la estática - se tratará de medir en términos del peso del cuerpo ${ }^{43}$. La interpretación es que, a lo largo de la caída, se produce instante a instante una acumulación de "momentos" iguales que, conservándose en el cuerpo, se liberan de golpe en el instante de la percusión. De ahi Galileo, en su póstuma 6.2 Jornada de los Discorsi (publicada en 1718), concluyó que la fuerza de percusión es indeterminada o infinita en relación con el peso. Su discípulo Torricelli, aún desconociendo la obra del maestro, la expuso casi en los mismos términos en su Lezioni Accademiche que, pronunciadas ante la Academia de la Crusca en 1642-43, sólo se publicarán en 1715. Según Torricelli, en cada instante de tiempo la gravedad engendrará sobre un cuerpo un «momento» igual a su

pression of every point, with their planes of contact etc.: and how all these are varied every moment during the time of contact by the more or lesse relenting softnesse or springynesse of these bodies and their various slidings. And also what effect the air or other mediums compressed betwixt the bodies may have». HERIVEL (1965), V, pags. 213-214. (La escritura de esta parte se debe a la mano del que fuese amigo y companiero de habitación John Wickins). También en Hall y Hall (1978), págs. 162-163.

42 Véase Solts (1987), esp. págs. 55-59.

43 Westrall (1971), Costabel (1983), De Gandt (1987) y De GANDT (1995) se ocupan de esta tradición. 
peso. Si el cuerpo está en reposo, digamos, sobre una tabla, ésta la anulará, la matará instante a instante. Pero en la caída libre los momentos se acumulan, liberando en la percusión un momento igual a tantas veces el peso como instantes se cuenten en el intervalo de tiempo de caída. Siendo éstos infinitos, el peso y la fuerza de percusión serán estrictamente inconmensurables a menos que el choque requiera un cierto tiempo ${ }^{44}$. Otros autores presentaron concepciones similares respecto a la inconmensurabilidad de los dos tipos de fuerza. Tal es el caso de Descartes al comparar en su correspondencia la fuerza empleada para sostener un peso con la necesaria para elevarlo hasta cierta altura ${ }^{45}$. En su De vi percusionis (1667), Giovanni Borelli expresó que la relación del peso a la fuerza del impetus es como la de la línea a la superficie ${ }^{46}$. Más tarde, en 1695, Leibniz incluirá la gravedad o fuerza centrípeta entre las "fuerzas muertas", solicitaciones al movimiento que guardan respecto de las "fuerzas vivas" la misma razón de las cantidades infinitesimales a las finitas ${ }^{47}$.

En las concepciones mecánicas de un éter que actúa por impulsos, a mayor número y sutileza de las partículas etéreas que ejercen su acción corresponderá, en general, una trayectoria tanto más próxima a una curva rigurosa; y todos los filósofos naturales de la época coincidían en atribuir a los componentes del éter la mayor de las sutilezas. Si las partículas del éter, como suponía Descartes, son duras, o si en todo caso el lapso de la interacción de cada una de ellas con la materia grosera es muy breve, cada impulso se puede considerar instantáneo, y la trayectoria del cuerpo sometido a estas innumerables acciones se podrá representar por una línea poligonal, de lados infinitesimales si el número de los impulsos que sufre el cuerpo en un intervalo de tiempo dado es muy grande. Concebida de esta manera la trayectoria, la curva rigurosa no serfa más que una aproximación a la verdadera trayectoria poligonal, al contrario de lo que han sostenido hasta ahora los estudiosos del modelo de impactos.

Newton se reservó muy mucho de expresar sus opiniones sobre estas materias, de modo que cualquier interpretación contiene necesariamente una buena

44 Vease De GANDT (1987), págs. 54-77; la traducción al francés del texto de Torricelli, en págs. 207-224.

45 Véase WestFall (1971), págs. 72 ss., especialmente la primera cita en la pág. 76.

46 Ibid, págs. 222 y 229.

47 Véase COSTABEL (1983), págs. 23-29. El texto del "Specimen dynamicum", publicado en las Acta Eruditorum de 1695, se reproduce en las págs. 96-101. En su Essay de Dynamique (1692) la razón es la del punto a la línea. Ibid, pág. 93. 
dosis de especulación. Pero de lo anterior se ve que las dos conceptualizaciones de la fuerza que sugiere Westfall no son necesariamente incompatibles. Todavía más, en una primera etapa, cuando Newton concibe a las curvas rigurosas como líneas poligonales infinitangulares, cuanto menos a nivel del cálculo matemático la compatibilidad es total. En una segunda etapa, cuando Newton se muestre insatisfecho con el fundamento geométrico de los infnitesimales, llegará a la curva rigurosa como límite de una aproximación poligonal con creciente número de lados. Tal proceso de paso al límite supone la eliminación de las fuerzas motrices instantáneas impresas en cada vértice y con él un cambio en las dimensiones de la fuerza, que de discreta pasa a continua. Esto supone, asimismo, renunciar a la ontología de impulsos que explicaría la acción del éter. Anteriormente se mencionó que el tránsito entre las dos concepciones de Newton del cálculo se díb, más o menos, a juzgar por los contenidos de sus manuscritos, hacia 1684. Precisamente de 1680 data el experimento de Newton con péndulos, destinado a detectar la resistencia que pudiese ofrecer el éter al movimiento de los cuerpos. Sus resultados negativos, en coincidencia con otras consideraciones, le llevaron a rechazar la existencia de este medio, postura que sostuvo cuando menos hasta la segunda edición inglesa de los Principia en $1713^{48}$.

El modelo poligonal y la curva rigurosa

Como remate de las consideraciones efectuadas en el apdo. anterior e introducción al tratamiento de Newton del movimiento circular, analizaremos brevemente, desde un punto de vista geométrico, lo que sucede en la modelización de un choque físico mediante un impulso instantáneo, así como en el tránsito desde el modelo poligonal a la curva rigurosa.

En la Fig. 1, la curva $A B C$ representa la variación de la intensidad de la fuerza en un choque elástico con deformación que tiene lugar durante un intervalo de tiempo $\Delta t$. El área bajo la curva representa la fuerza total, proporcional al producto de la intensidad por el intervalo de tiempo. Al cabo de este mismo intervalo, la fuerza total es la misma si suponemos que el choque se ha efectuado con la intensidad media $I_{m}$, siendo el área del paralelogramo $A D E C$ igual al 


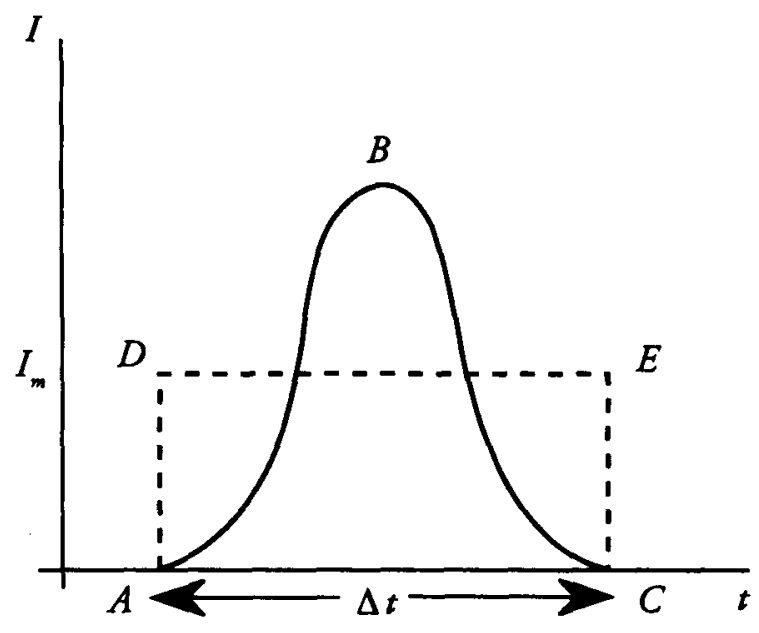

Fig. 1

área bajo la curva $A B C$. Así, la intensidad $I_{d}$ del impulso instantáneo equivalente es la medida del área del paralelogramo $A D E C$. Mientras $\Delta t \rightarrow 0$, cuando aún el paralelogramo es una superficie, existe un impulso instantáneo asociado, que es cada vez más pequefio. En el límite, tal impulso desaparece, y el cambio en la cantidad de movimiento es, asimismo, nulo. Así que, si queremos que en este límite la fuerza total sea igual a la de un impulso instantáneo, entonces no tendremos que mantener constante $I_{m}$, sino el área del rectángulo. Dividir $\Delta t$ por dos, esto es, dividir su base por la mitad, equivale a duplicar por dos su altura, $I_{m}$. El proceso de división se puede proseguir indefinidamente; en el límite, $I_{m}$ es una línea infinita - un infinito actual-y se halla más allá de toda medida. Y, todavía, podríamos decir que la fuerza total sigue siendo la misma en ambos casos, pues se ha mantenido así durante todo el proceso ${ }^{49}$. Si bien, estrictamente hablando, en $\Delta t=0$ se ha tornado indeterminada, y tal valor sólo se le puede atribuir en virtud de un cierto principio de continuidad ${ }^{50}$. Se ve

49 Este razonamiento no tiene por qué ser ajeno a la época. Fue precisamente el camino seguido por Nicolas Oresme para mostrar que si toda la cualidad de un cuerpo se hallase en un punto, esta sería infinitamente intensa; y, todavia, la cualidad total, al no haber variado a lo largo del proceso, seguiría siendo finita. El fragmento de Oresme se recoge en CLAGETT (1968), pág. 289.

50 En su teoría de las razones primeras y últimas, Newton admitirá que en límite las magnitudes nacientes o evanescentes no existen, aunque afirma que todavía subsistirá la razón entre ellas que se ha mantenido a lo largo del intervalo. 
que esto sucede porque, aunque en ambos casos las fuerzas totales son comparables, las intensidades de un impulso instantáneo y de una fuerza continua no tienen la misma dimensión. De (5) y (6) se ve que, en términos modernos, las dimensiones físicas de la fuerza total son $\mathrm{MLT}^{-1}$, las de la intensidad instantánea $I_{d}$ son $\mathrm{LT}^{-1}$ (una velocidad) y las de la intensidad continua $I_{c} \circ I_{m}$ son $\mathrm{LT}^{-2}$ (una aceleración).

En el modelo de impactos instantáneos, la curva rigurosa $A B C$ se ve aproximada por una línea poligonal, como muestra la figura 2. La acción continua de la fuerza centrípeta a lo largo del arco $B C$, recorrido en un tiempo $\Delta t$, se sustituye por un impulso instantáneo aplicado en $B$, de tal magnitud que, componiéndose con el movimiento inercial $B D$ que hubiese seguido el cuerpo de no actuar sobre él esta fuerza en el mismo tiempo en que se emplea en recorrer el tramo $B C$, lo lleva al punto $C$. El tramo por la cuerda $B C$ se recorre con movimiento uniforme, y la fuerza del impulso en $B$ se mide por el cambio en el movimiento que produce, medido por la longitud $D C=B E$ recorrida en $\Delta t$. La "fuerza total" de la fuerza continua en el arco $B C$ es así igual a la "fuerza total» del impulso en $B$.

Siendo $B$ y $C$ puntos fijos sobre la curva rigurosa, reducir el tiempo entre impactos equivale a aumentar el número de ellos. De modo que cuando $\Delta t \rightarrow 0$, el número de impactos entre $B$ y $C$ tiende a infinito. Con referencia a la figura 1 , esto equivale a hacer equivalente la fuerza continua entre $A$ y $C$ que aquí podríamos considerar constante sin perjuicio del argumento- no a un solo impacto instantáneo en $A$, sino a un número creciente de impactos instantáneos entre $A$ y $C$. Con referencia a la fig.1, cuando el número de particiones de $\Delta t$ se convierte en potencialmente infinito, el rectángulo $A D E C$ resulta de la suma de un número potencialmente infinito de rectángulos de altura $I_{m}$ y base infinitesimal $d t$. Supongamos ahora que este infinito potencial se actualiza, esto es, que se alcanza el límite y $\Delta t$ es rigurosamente cero. El número de impactos instantáneos entre los puntos fijos $B$ y $C$ de la fig. 2 se ha convertido en un infinito actual; los rectángulos de base infinitesimal se han convertido en líneas, de modo que el rectángulo $A D E C$ se ha convertido en todas las líneas perpendiculares a $A C$ y de altura $I_{m}$ trazadas desde todos y cada uno de los puntos del segmento $A C$. La fuerza total, que antes se entendía como la suma del número potencialmente infinito de rectángulos de base infinitesimal, ahora se ha vuelto indeterminada. Todavla podriamos decir que la suma de las infinitas líneas de longitud $I_{m}$ es precisamente igual, o cuanto menos equivalente, al rectángulo $A D E C$ cuya área - finita- mide la intensi- 
dad $I_{d}$ del impulso instantáneo asociado. Pero esto equivale a vulnerar el postulado de homogeneidad de Euclides; de modo que los indivisibles se sustituyeron por los infinitesimales, y con ello nació el cálculo.

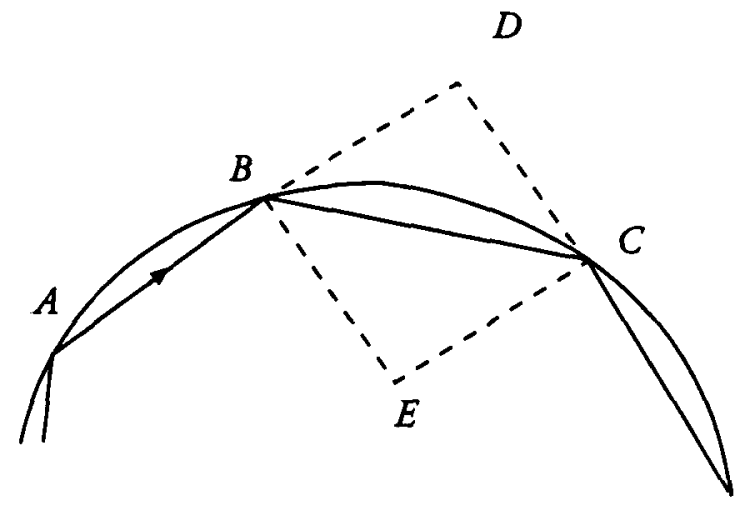

Fig. 2

El primero de los pasos, cuando aún el rectángulo resulta de la suma de una infinidad de rectángulos de base infinitesimal, corresponde a la primera versión del cálculo de Newton. El segundo, en el que los rectángulos se desvanecen en líneas en el paso al límite, corresponde a la formulación, pese a lo dicho no menos, sino más rigurosa, y más ciertamente fiel al espíritu de la geometría clásica, del método de las razones primeras y últimas.

Como veremos en el próximo apartado, en 1665 Newton estudió el movimiento circular aplicando este modelo de impactos instantáneos. Pero algún tiempo después, frente a esta aproximación poligonal, estudió este movimiento mediante otra que se ha calificado de "parabólica", y que se basa de partida en la curva rigurosa. En la misma entrada del "Waste Book" en la que Newton discute el impacto elástico de dos cuerpos deformables comparándolo con el de dos cuerpos duros, y estudia el movimiento circular a través del movimiento de una bola en el interior de un cilindro, se ocupa del movimiento del centro de masas - él lo llama "centro de movimienton- de un cuerpo. Había mostrado que, en el caso de un movimiento en línea recta, o de la combinación de éste con otro de rotación en torno al mismo centro, dicho centro tendrá la misma velocidad y determinación que el cuerpo. A continuación indicó que, cuando la trayectoria es 
una curva, sucede lo mismo que en el caso del movimiento rectilíneo, pues:

"(...) se puede concebir que una línea curva consiste en un número infinito de líneas rectas. $O$ bien en cualquier punto de la línea curva el movimiento puede concebirse sobre la tangentes ${ }^{51}$.

En principio, los dos casos no son equivalentes. Como ya se vio anteriormente, en el primero la velocidad es una "verdadera" velocidad, recorriéndose cada línea infinitesimal en un intervalo infinitesimal de tiempo aun cuando, a nivel finito, el movimiento del cuerpo sea acelerado. En el segundo caso la velocidad es puntual, instantánea, una mera potencialidad que sólo se puede medir por el comportamiento inercial que hubiese tenido el cuerpo si en tal punto se hubiese liberado de sus ligaduras.

Además, en ambos casos no coincide la dirección de la tangente en un punto. En la fig. 3 se ve que, en el caso poligonal, el cuerpo, abandonado a sí mismo, seguiría la línea $A C$; en la concepción de una curva como una línea poligonal infinitangular, la tangente se definió como la prolongación de la línea infinitesimal que une dos puntos infinitamente próximos. Mientras que

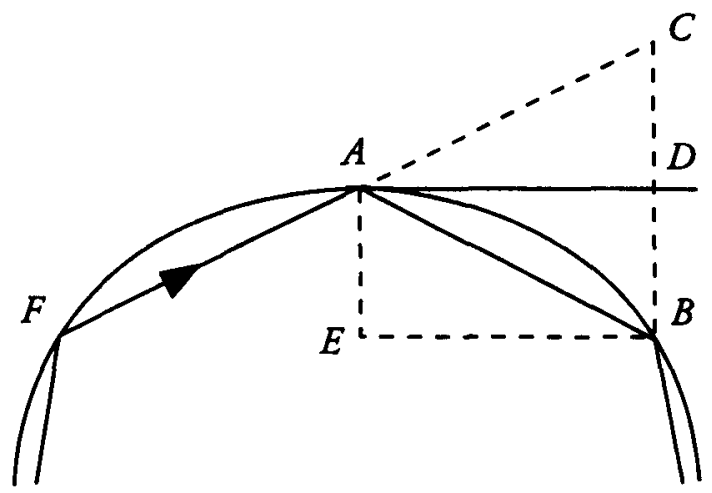

Fig. 3

S1 *(..) a crooked line may de conceived to consist of an infinite number of streight lines. Or else in any point of the crooked line the motion may bee conceived to be on in the tangent." HeRIVEL (1965), Iid, pág. 145. 
la tangente a la curva rigurosa sería $A D$. Si se calculase la fuerza centrípeta por la desviación $C B$, o la $D B$, de la trayectoria inercial, entonces los resultados diferirian en un factor de dos, pues se ve que $C D=D B^{52}$.

Pero todavía hay otra consideración. En el modelo poligonal, el movimiento de $A$ a $B$ resulta de la composición del movimiento inercial $A C$ y del impulso de la fuerza centrípeta en $A$, que durante el mismo intervalo de tiempo que invertirla el cuerpo en recorrer $A C$ lo obliga a recorrer $C B$ con movimiento uniforme. Por su parte, la aproximación parabólica parte de la curva rigurosa, y el movimiento de $A$ a $B$ resulta de la composición del movimiento inercial $A D$ y de la desviación $D B$ producida por una fuerza centrípeta que, al ser el arco $A B$ infinitesimal, se supone constante, y por tanto se recorre con movimiento uniformemente acelerado. La combinación de ambos movimientos aproxima el arco $A B$ por un segmento de parábola, aplicando las conclusiones de Galileo sobre el movimiento de proyectiles. Además, Galileo probó que, en un intervalo de tiempo dado, un cuerpo con movimiento uniformemente acelerado partiendo del reposo recorre la mitad de la distancia que cubrirla en el mismo intervalo de tiempo moviéndose con una velocidad constante igual a la velocidad final adquirida en el caso anterior. De nuevo la diferencia supone un factor de dos ${ }^{53}$.

El caso de la disparidad de resultados entre Varignon y Leibniz en el cálculo de la fuerza centrifuga es representativo de la confusión que, en los primeros años del siglo XVIII, existía sobre estas cuestiones. Varignon consideró la tangente según $A C \mathrm{y}$ al tramo $B C$ recorrido con moivimiento uniformemente acelerado, mientras que Leibniz compuso el tramo $A D$ con un movimiento uniforme sobre $D B^{54}$.

¿Se puede interpretar la cita anterior de Newton en el sentido de que ya en ese momento era consciente de la existencia de dos modelos alternativos para el análisis del movimiento centrípeto?

Permítaseme hacer un inciso en este punto para aclarar que aqui estamos jugando con cuatro elementos: Dos concepciones de curva (polígono infinitangular o curva rigurosa) y dos procedimientos de análisis (mediante

52 Según esto, la figura de la pág. 57 de BRACKENRIDGE (1995), en donde se compara la aproximación poligonal con la parabólica, contiene el error de hacer coincidir la dirección de la tangente en ambos casos.

53 Hankans (1970), págs. 225-228.

54 ArTON (1989), pág. 217. 
infinitesimales o mediante límites). El método de límites sólo es aplicable a la curva rigurosa o, por mejor decir, sólo termina en la curva rigurosa, pues dentro de tal proceso la curva poligonal sólo representa un paso muy próximo todo lo próximo que se quiera - a la curva rigurosa. El dominio del método infinitesimal, sin embargo, es en la época el de la curva poligonal infinitangular. Bien porque todas las curvas rigurosas se identifiquen con tales, bien porque la curva rigurosa se analice en tales términos como tal en su conjunto o en las vecindades de un punto ( $\mathrm{y}$ así, por ejemplo, el elemento "riguroso" de arco se puede sustituir por la cuerda). Naturalmente, toda esta situación no es sino consecuencia de los problemas de fundamentación que el cálculo infinitesimal encontró en la época, particularmente en el caso de su interpretación geométrica. Hoy en día, desde el rigor suministrado por el análisis no estándar, la línea poligonal infinitangular y la curva rigurosa se confundirían estrictamente porque, por decirlo así, los infinitesimales están dentro de los puntos de la curva rigurosa; y ambos métodos coinciden.

Tal como se aplicaron en el contexto de la época, ambos procedimientos llevaban a resultados correctos siempre y cuando - como fue el caso de Varignon y Leibniz- no se entremezclasen. Como se ha dicho, Newton pudo haber entendido el problema, y con ello lograr una buena comprensión de las cuestiones de fundamentación — del lado de la geometría- que el análisis tenía en ese momento. No sólo el hecho de que no confundiese ambas interpretaciones, sino también y muy especialmente su tránsito desde una teoría de infinitesimales a otra fundamentada en los límites, abogan por tal interpretación.

\section{El análisis del movimiento circular}

En la que se supone su primera investigación del movimiento circular de la que queda constancia, Newton estudio el movimiento de una esfera ceñida a moverse en el interior de una superficie cilíndrica, caso que consideró equivalente al de un cuerpo en rotación al extremo de un hilo o cuerda cuyo otro extremo está fijo en el centro de la circunferencia que describe:

"Si una esfera oc se mueve en la superficie cóncava esférica de la superficie cilindrica del cuerpo edf circularmente en torno al centro $m$, presionará sobre el cuerpo def pues cuando está en $c$ (suponiendo que su centro de movimiento describe el círculo bhc y que la línea cg es tangente a ese círculo en $o$ ) se mueve hacia $g$ o la determinación de su movimiento es hacia $c$ 
[sic., léase g], por lo que si en ese momento el cuerpo edfdejase de impedírselo, se movería continuamente en la línea $c g(. .$.$) oblicuamente desde el$ centro $m$, pero si el cuerpo def se opone a esta tendencia manteniéndolo equidistante de $m$, esto se hace por un impedimento o reflexión continuo desde la línea tangente en cada punto del círculo $c b h$, pero el cuerpo edf no puede obstaculizar y curvar la determinación del cuerpo co a menos que presionen continuamente entre sim 55 .

$\mathrm{Y}$ añade que lo mismo se puede decir si el cuerpo describe un movimiento circular al extremo de un hilo o cuerda. De modo que, si no fuese por la superficie cilíndrica, en un punto cualquiera de su trayectoria, como el $c$, el cuerpo se movería siguiendo la tangente cg. Si se considera el movimiento de la bola 0

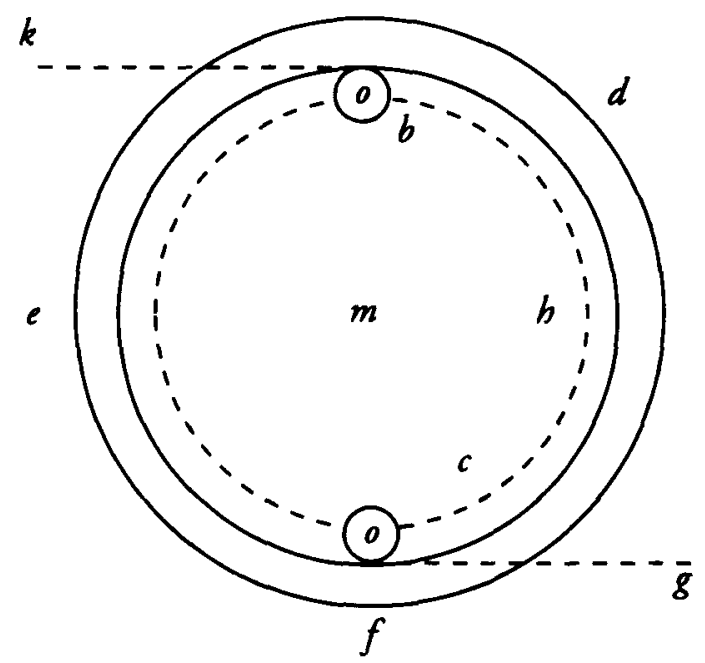

Fig. 4

55. If a spherae oc move within the concave shaeicall [sic] of cilindricall surface of the body edf circularly about the center $m$, it shall press upon the body deffor when it is in $c$ (supposeing the circle $b h c$ to be described by its center of motion and the line $c g$ a tangent to that circle at o) [it] moves towards $g$ or the determination of its motion is towards $c$ [sic] therefore if at that moment the body efd should cease to check it it would continually move in the line co $(2 x .1,2)$ obliqly from the center $m$, but if the body defoppose it selfe to this endeavour keeping it equidistant from $m$, that is done by a continued checking or reflection of it from the tangent line in every point of the circle $c b h$, but the body edf cannot check and curbe the determination of the body co unless they continually presse upon one another.» HERIVEL (1965), II, págs. 146-147. 
en media revolución, entonces la "resistencia» del cilindro, igual a la "presión» que actúa sobre 0 , es capaz de destruir su movimiento de $c$ a $g$ y generar otro opuesto de $b$ a $k$. De modo que la fuerza por la que $o$ tiende a apartarse del centro en esta media revolución es "más del doble» de la fuerza capaz de generar o destruir su movimiento ${ }^{56}$.

En unas anotaciones situadas al comienzo del "Waste Book", pero que consideraciones de consistencia interna sugieren posteriores al anterior tratamiento del movimiento circular, Newton empleó por primera vez el modelo de impacto. Imagina una bola $b$ que recorre una trayectoria circular, y escribe:

«Si la bola $b$ gira alrededor del centro $n$ la fuerza por la que tiende a apartarse del centro $n$ engendraría tanto movimiento en un cuerpo como el que hay en $b$ en el tiempo en que el cuerpo $b$ recorre la longitud del semidiámetro $b n$. [así si $b$ se mueve con un grado de movimiento atravesando $b n$ en un segundo de una hora, entonces su fuerza desde el centro siendo impresa continuamente (como la fuerza de gravedad) sobre un cuerpo durante un segundo generará un grado de movimiento en ese cuerpo]. O bien la fuerza desde $n$ en una revolución es a la fuerza de movimiento del cuerpo como: periferia: radio" 57 .

Casi toda la segunda parte de la cita está entre corchetes, un procedimiento alternativo a la tachadura empleado por Newton para cancelar un texto ${ }^{58}$. tencia del aire.

El movimiento de $b$, producto de su masa por su velocidad orbital (escalar), es siempre el mismo, pues el movimiento circular se supone uniforme. Lo que dice Newton es que la fuerza centrifuga, actuando linealmente durante el mismo tiempo que invierte $b$ en describir un arco de circunferencia igual a su radio, engendrarf́a tanto movimiento como el de $b$. O bien, si se considera una

56 HeRTVEl (1965), IId, págs. 146-147.

57 If the ball $b$ revolves about the center $n$ the force by which it endeavours from the center $n$ would beget soe much motion in a body as there is in $b$ in the time that the body $b$ moves the length of semidiameter $b n$. [as if $b$ is moved with one degree of motion through $b n$ in one seacond of an hower then its force from the center $n$ being continually (like the force of gravity) impressed upon a body during one second it will generate one degree of motion in that body.] Or the force from $n$ in one revolution is to the force of the bodys motion as :: periph : rad.» Ibid, IIa, pág. 129.

58 Véase BRACKENRIDGE (1995), pág. 273, nota 8. 
revolución completa, esta fuerza centrífuga, actuando linealmente, engendraría en un cuerpo en una duración igual al período un movimiento que sería al movimiento (orbital) de $b$ como la circunferencia al radio, esto es, $2 \pi$ veces mayor.

Para probar esto, Newton imagina un cuadrado circunscrito a la trayectoria circular, y a un cuerpo recorriendo una trayectoria cuadrada al rebotar en los puntos $a, b, c, d$. Supongamos al cuerpo desplazándose de $a$ a $b$, cuando se produce el impacto en $b y$, a consecuencia del mismo, el cuerpo recorre el tramo $b c$, este desplazamiento, $b c$, resulta igual a la composición del que se habria producido si el choque no hubiese tenido lugar, by, y otro, efecto del choque, $y c=b d$, recorridos cada uno de ellos en el mismo intervalo de tiempo que invierte el cuerpo en cubrir $b c^{59}$. De modo que la "fuerza o presión" de $b$ sobre fg en la reflexión es a la "fuerza del movimiento" de $b$ como:

$$
\frac{y c}{a b}=\frac{2 f a}{a b}=\frac{a b}{f b}
$$

pues $a b^{2}=f a^{2}+f b^{2}=2 f a^{2} ;$ y dividiendo ambos miembros por falab se llega a la expresión final. En un circuito completo, la suma de las fuerzas de reflexión en los vértices es a la fuerza del movimiento del cuerpo como la suma de los lados del cuadrado al radio del ćrculo. Acto seguido, Newton generaliza el resultado al círculo mismo:

"Por el mismo procedimiento si el Globo $b$ fuese reflejado por cada lado de un poligono circunscrito de 6, 8, 12,100, 1000 lados, etc., la fuerza de todas las reflexiones es a la fuerza del movimiento del cuerpo como la suma de aquellos lados al radio del círculo en el que se hallan circunscritos. $Y$ así, si el cuerpo fuese reflejado por los lados de un polígono equilátero circunscrito de un número infinito de lados (i.e., por el ćrculo mismo) la fuerza de todas las reflexiones es a la fuerza del movimiento del cuerpo como todas aquellas caras (id est el perímetro) al radion ${ }^{60}$.

59 Las líneas de trazos by, yg, y bd no aparecen en la figura original.

60 «(...) by the same proceeding if the Globe $b$ were reflected by each side of a circumscribed polygon of $6,8,12,100,1000$ sides etc. the force of all the reflections is to the force of the bodys motion as the sume of those sides to the radius of the circle about which they are circumscribed. And so if [the] body were reflected by the sides of an equilaterall circumscribed polygon of an infinite number of sides (i.e. by the circle it selfe) the force of all the reflections are to the force of the bodys motion as all those sides (id est the perimeter) to the radius.» HERIVEL (1965), Ila, pág. 130. 
Esta demostración ha dado algún trabajo a los historiadores. Herivel observó que el procedimiento seguido por Newton no dejaba de ser problemático. Pues en el polígono de infinitos lados la suma permanece finita mientras que las fuerzas individuales tienden a cero; y además, dado que su dirección varía en cada punto, a Herivel el sentido físico de esta suma le parece dudoso ${ }^{61}$. Más recientemente, Erlichson ha dado respuesta a estas objeciones. La fuerza centrífuga es igual a la fuerza motriz total, y es la suma escalar de los efectos de las fuerzas individuales de reflexión. Asimismo, en el límite dichas fuerzas individuales se anulan, pero no la fuerza centrípeta en un punto. Y, aunque senala que aquí Newton no calcula dicha fuerza, apunta que su valor finito se debe a que es medida por el cambio en la cantidad de movimiento dividido por el intervalo de tiempo ${ }^{62}$. Por lo que se ha visto anteriormente, la interpretación de Erlichson es acertada. Pero pienso que los términos en los que la expone son menos apropiados que los que se recogen en este trabajo. El mismo Erlichson señala que, para entender el trabajo de Newton, aunque los símbolos y conceptos modernos puedan constituir una ayuda, hay que hacer un esfuerzo por entender los conceptos de una mecánica que Newton formuló en el siglo XVII 63. Todavía creo que debería haber llevado algo más lejos este esfuerzo de comprensión. Ha visto la centralidad que tiene para Newton lo que él denomina "cantidad motriz de una fuerza", y que aquí se denomina, más de acuerdo con la primera etapa de sus indagaciones mecánicas, como "fuerza total" 0 , si se quiere mayor precisión, "cantidad de fuerza total». Pero no ha visto - quizás no ha buscado- su origen y su justificación. Que las definiciones del «De gravitatione" que hemos examinado aquí recuerden a la vieja doctrina de la latitud de las formas tal como la interpretó Nicolas Oresme no significa tanto una oculta vinculación de Newton con ciertos pensadores medievales como el hecho de que constituía una teoría de la medida de cantidades variables que se empleaba en geometría. Y su aplicación al caso de una fuerza que, como la centrípeta, podía variar en magnitud y en dirección responde tanto a esta tradición como a los problemas, que más adelante discutiremos, relativos a la aplicación del paralelogramo de composición de movimientos a los efectos de una fuerza tal. Finalmente, Erlichson da un salto de veinte años e interpreta estos primeros estudios de Newton a la luz de la teoría de límites, pasando por alto

\footnotetext{
61 Ibid., págs. 9-10.

62 ERLiCHSON (1991a), pág. 845.

63 Ibid, pág. 843.
} 
la identificación del mismo Newton del círculo con un polígono de infinitos lados.

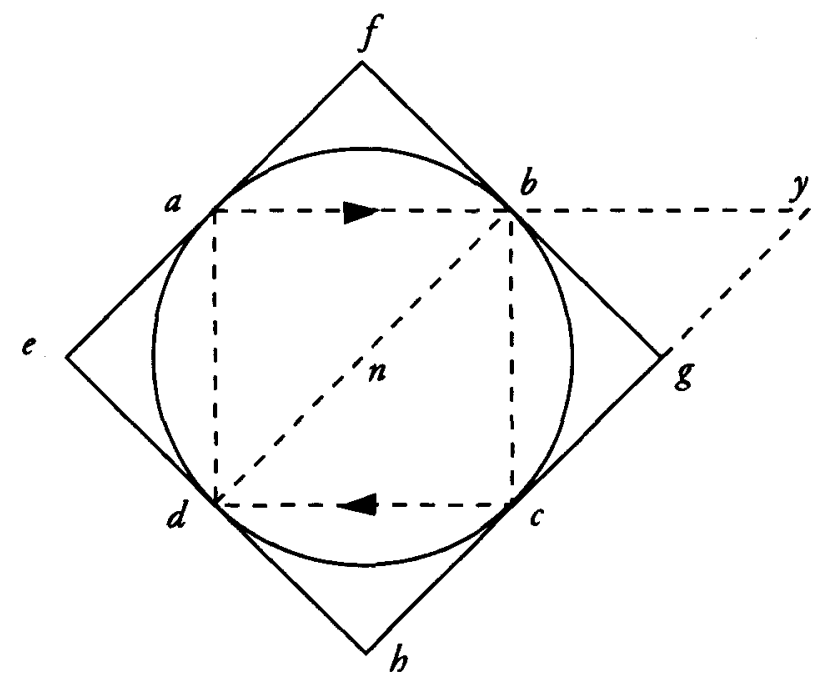

Fig. 5

D. T. Whiteside, al año siguiente de la publicación del estudio de Herivel, comentó esta demostración de Newton haciendo hincapié en las sutilezas que se presentaban al aproximar un círculo por un polígono tal, reiterando su interpretación en años posteriores. E.J. Aiton retomó esta misma cuestión. Erlichson respondió a ambos. Pero permítaseme dejar la discusión para más adelante, una vez reunidos más datos sobre la obra newtoniana.

Entre tanto, terminemos esta discusión historiográfica con la opinión de Westfall. Éste, como se ha comentado, señaló la distinta dimensionalidad de las fuerzas de impacto y la fuerza del movimiento circular, una distinción que según él Newton mismo habría admitido cuando, al tratar del caso de una bola moviéndose por el interior de un cilindro, habla de la "fuerza total» durante media revolución ${ }^{64}$. Señala que la ambigüedad entre estos dos modelos de fuerza, la basada en el impacto o la suministrada por el análisis de

64 Westrall (1971), pág. 351. 
Galileo, aparece ya en estos primeros escritos dinámicos, plagando la obra newtoniana hasta el final. E interpreta la fuerza que interviene en el movimiento circular en sentido moderno. De modo que, para una revolución, $F / m \nu=2 \pi r / r$, o bien $F=2 \pi m v$. Dividiendo por el tiempo de una revolución $(2 \pi r / v)$, se obtiene el valor del $m v^{2} / r$, la fuerza desde el centro en cada instante $^{65}$. Erlichson niega que las distintas dimensiones de estas fuerzas supusiesen un problema para Newton. Está en lo cierto al decir que la obtención de la fuerza en un punto no entra en la discusión de Newton, y que por consiguiente en este estudio no entran en juego fuerzas con distintas dimensiones (y más adelante veremos por qué). Esta división por el período, dice, "es de Westfall, no de Newton" "66. Igualmente podemos decir que el paso al límite que en esta demostración convierte al polígono en un círculo riguroso es de Erlichson, no de Newton.

En relación con los dos modelos para la conceptualización de la fuerza, hay que decir que finalmente el primero, basado en la teoría de Descartes y en las necesidades de los atomistas, acabó perdiendo fuerza ante el segundo. Leibniz se deshizo de los choques instantáneos, desmarcándose de Descartes y, si Newton nunca renunció a sus átomos duros, los escondió bajo la doble alfombra de unas partículas de distintos órdenes de composición superior, y una notoria escasez de materia en el mundo comparada con sus espacios vacíos. Finalmente, para él los choques entre cuerpos duros dejarán de ser elásticos. Cualquiera que fuese lo que sucediese a nivel microscópico entre las partículas o átomos constituyentes del mundo, inalcanzable para la observación y la experimentación, los fenómenos naturales a nivel macroscópico parecían exhibir siempre fuerzas continuas, siquiera fuese durante brevisimos intervalos de tiempo.

Newton abordó de nuevo el estudio del movimiento circular en algún momento anterior a 1669 , pero que cabe suponer posterior a su primera aproximación por el modelo poligonal ${ }^{67}$. En la fig. 6, supuso que el cuerpo se movía sobre la circunferencia $A D E$, girando en el sentido $A D$. Consideró que $A D$ se describe en un tiempo muy pequeño ("minutissimum»), durante el cual la fuerza centrifuga es tal que lo separaría de la circunferencia la distancia $D B$. Esta distancia se recorrería con movimiento acelerado:

65 Ibid., pág. 355. Asimismo WeSTFAll (1980), págs. 148-150.

66 ERLICHSON (1991a), pág. 845.

67 Herivel (1965), IVa, págs. 192-195. También en Correspondence, Vol. I, págs. 297- 303. 


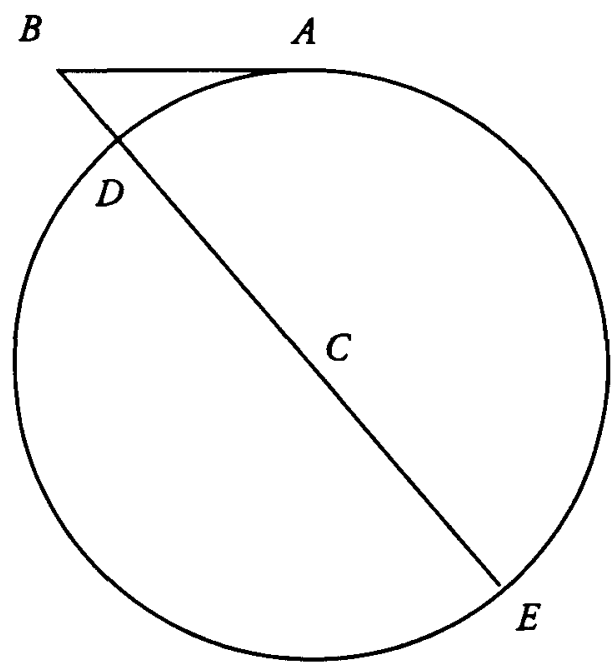

Fig. 6

"(...) este conatus, si actuase en línea recta y continuamente a la manera de la gravedad, impulsaría a los cuerpos a través de distancias que son como el cuadrado de los tiempos (...) ${ }^{68}$ ".

Si $t$ es el tiempo invertido en recorrer $B D$, Newton busca la distancia - llamémosla $X$ - que recorrería en el período $T$ de una revolución:

$$
\frac{X}{B D}=\left\lceil\frac{T^{2}}{t^{2}}\right\rceil=\frac{A D E A^{2}}{A D^{2}}
$$

Por Euclides 3.36 se cumple que $B E / B A=B A / B D$. Newton supone que la diferencia entre $B E$ y $D E$, y también entre $B A$ y $D A$, es infinitamente pequenia, lo que le permite sustituir y escribir que $D E / D A=D A / D B$, de modo que $D A^{2}=D E ! D B$, y:

$$
\frac{A D E A^{2}}{A D^{2}}=\frac{A D E A^{2}}{D E \cdot D B}
$$

68 (...) hic conatus corpora, si modo in directum ad modum gravitatis continuo urgeret, impelleret per spatia quae forent ut quadrata temporum (...).» Ibid., pág. 193. 
obteniéndose:

$$
X=\frac{A D E A^{2}}{D E}=\frac{\pi^{2} \cdot D E^{2}}{D E}=\pi^{2} \cdot D E
$$

y en consecuencia -este resultado aparece como corolario- los conatus desde los centros de diversos círculos son como sus diámetros divididos por los cuadrados de los períodos, lo que podríamos escribir:

$$
F_{c} \propto \frac{D}{T^{2}}
$$

Newton no especifica cómo se da el paso de (12) a (13), y los autores que han comentado este manuscrito no van mucho más allá de indicar que es una consecuencia de la ley de Galileo para el movimiento uniformemente acelerado. Según Herivel,

«Si la distancia recorrida en el tiempo $T$ de una revolución completa es proporcional a $R$, entonces por la ley $t^{2}$ la distancia recorrida en la unidad de tiempo (la base material de comparación de diferentes movimientos) será proporcional a $R / T^{2}{ }^{269}$.

$\mathrm{Si}$, de acuerdo con Westfall, el empleo de la ley de Galileo supone la interpretación implícita de su cinemática en términos dinámicos, entonces el paso es importante ${ }^{70}$. Más adelante, en la Prop. 3 del de Motu, Newton determinará la fuerza centrípeta en un punto aplicando la siguiente consideración; si $Q R$ representa la desviación,

“(...) la pequeña línea $Q R$ en un tiempo dado es como la fuerza centrípeta, $y$ la fuerza dada es como el cuadrado del tiempo, $y$ por lo tanto cuando ningu-

69 Ibid., pág. 198, nota 6. Véase asimismo la pág. 12. Según Brackenridge: «Recuérdese que $X$ era la distancia lineal atravesada en un tiempo igual al periodo de revolución $T$ bajo una fuerza lineal constante dirigida hacia el centro en dirección radial igual en magnitud a la tendencia [endeavour] a separarse del centro. Por consiguiente, la magnitud de la fuerza es inversamente proporcional al cuadrado del tiempo $T$ y directamente proporcional a $X(.$.$) .*$ BRACKENRIDGE (1995), pág. 62.

70 WestFall (1980), pág. 151. 
no de ellos está dado es como la fuerza centrípeta y el cuadrado del tiempo conjuntamente (...)m ${ }^{71}$.

En las conferencias de Motu, así como en la primera edición de los Principia, Newton justifica que la desviación es proporcional a la fuerza centrípeta remitiendo a la Ley II. Dado que a Herivel dicha Ley le parece una ley de impacto, opina que la justificación de Newton es poco satisfactoria, y su pensamiento respecto de los dos tipos de fuerza, a las que denomina motriz y acelerativa, confuso ${ }^{72}$. Como se ha argumentado anteriormente, en la interpretación que aquí adoptamos no hay confusión. La Ley II alude a las fuerzas totales, instantáneas o continuas. Tratándose de una fuerza continua, por nuestra ec. (6) la fuerza total, al cabo del tiempo $t$, es:

$$
F_{T C}=\Delta(m v)=m I_{C}
$$

Como el cuerpo ha partido del reposo, el cambio en el movimiento es el movimiento final; por el teorema del grado medio, éste es igual al doble del movimiento con que el cuerpo hubiese recorrido ese mismo espacio - llamémosle $X$ - con movimiento uniforme ${ }^{73}$. Si llamamos $v_{f}$ a la velocidad final, $y$ $v_{m}=1 / 2 v_{f}$ a la velocidad «media»:

$$
F_{T C}=\Delta(m v)=m v_{f}=2 m v_{m}=2 m \frac{X}{t}=m I_{C} t
$$

de donde:

$$
I_{C}=\frac{2 X}{t^{2}}
$$

71 "(...) lineola $Q R$ dato tempore est ut vis centripeta et data vi ut quadratum temporis atque adeo neutro dato ut vis centripeta et quadratum temporis conjunctim, (...)". HERVEL (1965), IX, pág. 260. Nótese la similitud con nuestra interpretación de la Def. VII en el apartado sobre el impacto y la fuerza.

72 Ibid., pág. 22.

73 Particularmente, como es sabido, Galileo empleó esta conclusión de la teoría medieval de la configuración en su tratamiento del movimiento uniformemente acelerado, en el Teorema, Prop. I de la Jornada Tercera de sus Discorsi. 
y el espacio recorrido resulta:

$$
X=\frac{1}{2} I_{C} t^{2}
$$

Se ve que (16) tiene la misma forma que (13). De hecho esta deducción -que en la época se hubiese hecho empleando el lenguaje geométrico de las proporciones- no figura entre los manuscritos de Newton. Pero podría haber sido el camino seguido para traducir la ley de Galileo a términos dinámicos, y justificarla tanto la alusión citada anteriormente a la Ley II como su afirmación, en el Escolio a los "Axiomas o leyes del movimiento" de los Principia, de que Galileo descubrió la ley de caída de los graves y el movimiento parabólico gracias a las dos primeras Leyes y los dos primeros corolarios ${ }^{74}$.

En el manuscrito que contiene la deducción de la fuerza centrifuga que acabamos de comentar, Newton calcula su valor en el movimiento de rotación terrestre y lo compara con el de la gravedad ${ }^{75}$. Pero también es notable por otros aspectos. Uno de ellos tiene que ver con el sentido de la desviación. Newton parece seguir aquí a Descartes al considerar que la desviación es radial y el conatus, centrffugo. Sin embargo, en la aproximación al movimiento circular mediante el modelo de impacto vista anteriormente, las "fuerzas de reflexión" en los vértices son centrípetas. Según la interpretación clásica de Herivel y Westfall, Newton sólo habría mostrado una buena comprensión de la dinámica del movimiento circular a partir del de Motu (1684), y ésta sería su deuda hacia Hooke en la correspondencia de 1679, a la que aludiremos más adelante.

Según Herivel, en una primera etapa Newton habría seguido a Descartes al pensar que en el movimiento circular entraban en juego dos conatus separados: uno a lo largo de la tangente y el otro centrífugo en dirección radial. $\mathrm{Si}$, en un momento dado, el cuerpo rompiese la ligadura, el conatus centrífugo lo impulsaría a recorrer la distancia $D B$ en el pequeño intervalo de tiempo empleado en recorrer $A D^{76}$. Según Westfall, en 1679 Newton todavía consideraría al cuerpo

74 Dichos corolarios se refieren a la composición de movimientos y fuerzas. NEwTON (1726; 1972), págs. 64-65. Trad. de E. Rada, págs. 145-146.

75 Dando respuesta así al viejo problema de por qué, si la Tierra gira, los objetos no salen despedidos.

76 Herviel (1965), págs. 45-48, y págs. 12-13. Sobre el movimiento circular en Descartes véase SHEA (1993), págs. 387-393. 
que gira en estado de equilibrio entre dos fuerzas opuestas: la gravedad y la vis centrifuga ${ }^{77}$. La confusión se daría entre los puntos de vista desde dos sistemas de referencia. Uno, inercial, estaría - digamos- ligado al centro del movimiento. Visto desde él, dicho movimiento resultaría de la composición de un movimiento inercial por la tangente y otro debido a la acción de una fuerza centrípeta. El otro sistema de referencia estaría vinculado al cuerpo que gira, y desde él se consideraría que el cuerpo se encuentra en reposo entre dos fuerzas - centrípeta y centrifuga - iguales y opuestas. Más recientemente, Erlichson ha argumentado que Newton no confundió los puntos de vista de ambos sistemas ${ }^{78}$. Para afirmar esto se apoya, entre otras cosas - particularmente en una reconstrucción según el modelo de impacto de la carta de Newton a Hooke del 13 de diciembre de 1679- en el carácter centrípeto de los impulsos del modelo de impacto, en su percepción del principio de acción y reacción y en el hecho de que, en la demostración de más ariba, Newton buscaba calcular precisamente la fuerza centrifuga (igual, por otra parte, a la centrípeta).

Ciertamente, cuando Newton estudia el movimiento circular de la bola por el interior del cilindro, la interacción entre ambos está clara: la bola presiona al cilindro, y éste a la bola. Pero no está claro que Newton considere al cuerpo en equilibrio entre una fuerza centrífuga y otra centrípeta, y que obtenga aquélla anulando ésta; como tampoco que distinga bien la situación entre el sistema de referencia inercial y el ligado al cuerpo que gira, cosas ambas que afirma Erlichson. Éste es su supuesto de partida:

«Si el cuerpo A gira en un círculo $\mathrm{AD}$ hacia $\mathrm{D}$, su conatus desde el centro es tanto cuanto sería el que, en el tiempo $\mathrm{AD}$ (que supongo pequeńísimo) lo separase de la circunferencia la distancia DB: pues en ese tiempo recorrería dicha distancia si se moviese libremente por la tangente sin impedimentos a su conatuss 79 .

Éstos son términos estrictamente cartesianos. Tanto en $E l$ mundo como en los Principios de filosofia, Descartes analiza el movimiento circular descompo-

77 WestFall (1971), págs. 426-427.

78 ERLICHSON (1992).

79 «Corporis $\mathrm{A}$ in circulo $\mathrm{AD}$ versus $\mathrm{D}$ gyrantis, conatus a centro tantus est quantus in tempore $\mathrm{AD}$ (quod pono minutissimum esse) deferret a circumferentia ad distantiam DB: siquidem eam distantiam in eo tempore acquireret si modo conatu non impedito libere moveret in tangente AB.» HERIVLL (1965), IVa, pág. 193. 
niéndolo en tres: uno según la tangente, otro según el círculo (no impedido) y otro radial centrífugo, pudiéndose entender que la tendencia según la tangente se compone de la tendencia a moverse por el círculo y la tendencia centrifuga ${ }^{80}$. Esto mismo es lo que dice Newton en la cita anterior. En El mundo, Descartes pone el ejemplo de una piedra que gira retenida por una honda, y en los Principios le añade otros dos: el de una hormiga que se desplaza por una regla en rotación, y el de una bola que se mueve por el interior de un tubo hueco asimismo en rotación. Supone que la hormiga camina por la regla alejándose del centro, de modo que en cada momento está sobre la tangente (fig. 7). El movimiento de la hormiga, o el de la bola, irá incrementando su velocidad en cada momento, pues el esfuerzo por alejarse del centro se renueva en todos y cada uno de los instantes. Descartes no analiza este movimiento acelerado, pero Newton (y, por su parte, también Huygens) interpretará dinámicamente la ley de Galileo suponiendo que el conatus centrifugo constante, de poder actuar sin impedimento, produciría un movimiento uniformemente acelerado.

Otro problema tiene que ver con la dirección de la desviación. En su estudio del movimiento circular (publicado en 1690), Huygens se preguntó, en el caso de un cuerpo que gira atado a un hilo, cómo es que la tensión sobre el hilo tiene

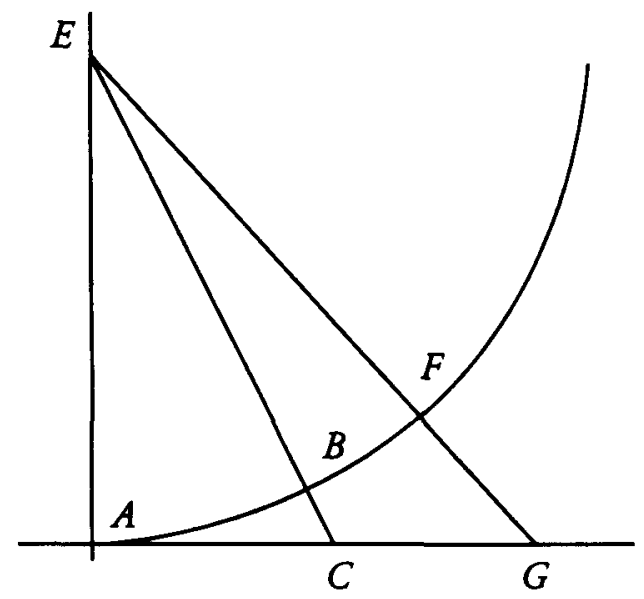

Fig. 7

80 Lo cual, desde luego, implica una cierta confusión respecto del principio de inercia rectilínea. Véase SHEA (1993), págs. 389 y 391. 
una dirección radial mientras que la tendencia del cuerpo es tangencial, perpendicular al radio ${ }^{81}$. La respuesta la encuentra analizando el problema desde un sistema de referencia ligado al cuerpo en rotación. Supongamos a un observador sobre el cuerpo que gira, el cual suelta una bola cuando se encuentra en $A$ (fig. 8). Desde el sistema inercial — digamos desde el centro $C$ del círculo- se ve a la bola desplazarse según la tangente, ocupando, a intervalos de tiempo iguales, las posiciones sucesivas $A_{l}, A_{2}, A_{3}$, etc., mientras que el observador, ligado al cuerpo que gira, se va desplazando y ocupando sucesivamente las posiciones $B_{l}$, $B_{2^{2}} B_{3^{2}}$ etc. Pasemos ahora al punto de vista del observador. Con el transcurso del tiempo, como hemos visto, éste ha ido dejando atrás el punto $A$ en el que soltó la bola, y al observador, que se considera a sí mismo inmóvil y fijo en $B_{4}$ le parecerá que, tras el primer intervalo de tiempo, el punto de lanzamiento habrá retrocedido a $B_{3}$, en el segundo intervalo a $B_{2}$ etc. De este modo, la trayectoria que verá el observador será una curva. En ella el segmento $A A_{1}$ tiene la misma longitud que el arco $A B_{1}$, el segmento $A A_{2}$ que el arco $A B_{z 2}$ etc. La curva, llamada evoluta, es la que trazaría el extremo $A_{4}$ de la tangente en $A$ si, manteniéndose tensa, se enrrollase en el círculo. En $B_{4}$ la tangente de la evoluta es el radio, de modo que ésta es la respuesta a la pregunta que se hizo Huygens.

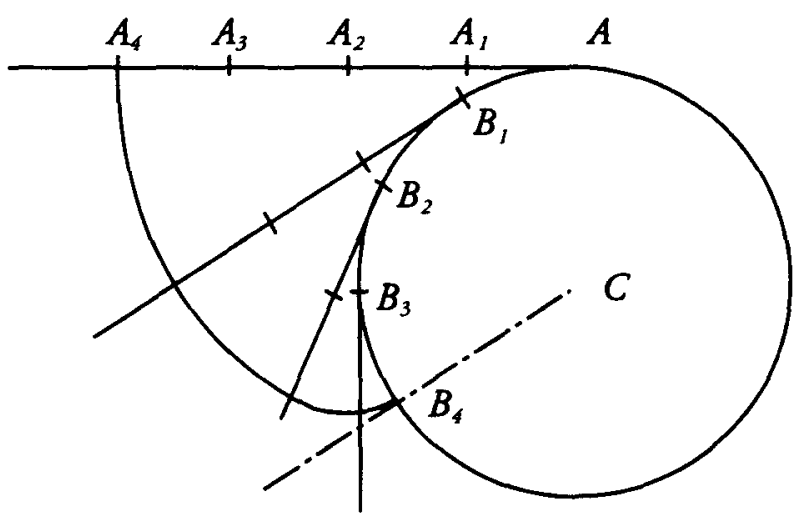

Fig. 8

81 Los resultados de Huygens, obtenidos en 1659, se publicaron sin demostración como apéndice a su Horologium oscillatorium (1673). Las demostraciones se publicarfan póstumamente en su *De vi centrifuga*, incluido en sus Opuscula posthuma (1703). El trabajo de Huygens se analiza en DE GANDT (1995), págs. 124-139. 
Retornando al sistema inercial, Huygens sustituyó la desviación por un arco de evoluta, al cual aproximó por una desviación rectilínea. Newton, por su parte, consideró directamente la dirección radial, sin mayores explicaciones; cabe suponer que aquí siguió fielmente a Descartes, calculando el conatus a partir del movimiento de la hormiga a lo largo de la regla, o de la bola a lo largo del tubo.

Una última cuestión atañe al empleo de la desviación como medida de la fuerza. Comentando la demostración, Herivel observa que Newton no hace al conatus proporcional a la desviación $D B$, sino que sólo dice que el conatus es de tal magnitud que llevaría al cuerpo a través de la distancia $B D$ en el tiempo correspondiente a $A D$. Y prosigue:

«Naturalmente, tal identificación hubiese sido falsa, puesto que el conatus es finito mientras que la desviación $\mathrm{DB}$ es muy pequeha” ${ }^{82}$.

Tampoco -escribe- hace ninguna consideración acerca de la verdadera relación entre desviación y conatus, al ser éste constante, es posible deducir la distancia recorrida en cualquier tiempo finito dada la distancia recorrida en el tiempo correspondiente a $A D$.

"Esto bastaba para comparar la magnitud del conatus con la de cualquier otra fuerza constante, tal como la fuerza de gravedad, o el conatus de algún otro movimiento circularn ${ }^{83}$.

Y finaliza diciendo que este método de comparar las fuerzas por las distancias recorridas en un intervalo finito de tiempo deja de ser aplicable en los casos en que varía la magnitud de la fuerza.

En realidad, como hemos visto, la fuerza centrifuga es un conatus, una fuerza impedida, de modo que de hecho no produce ningún movimiento, ningún desplazamiento del cuerpo. A diferencia de la fuerza centrípeta, que se compondrá con el movimiento inercial por la tangente para dar lugar a una trayectoria curva, produciendo un verdadero desplazamiento del cuerpo, y por tanto pudiéndose medir por el cambio en el movimiento que realmente ha

82 HeRIVEl (1965), pág. 13.

83 Ibid. 
producido, el conatus sólo se puede medir por el cambio en el movimiento que hubiese podido producir.

Por otra parte, no produciendo verdadero movimiento, el conatus es sólo una intensidad, no tiene cantidad motriz. Si la tuviese, como es la suposición de Newton, entonces sería ésta la que, dado el intervalo de tiempo, vendría medida por la desviación. Y, si ésta es pequefía o infinitesimal, también lo será proporcionalmente la cantidad motriz.

Cuando, más adelante, Newton emplee la fuerza centrípeta y trate el problema sobre la curva rigurosa, se enfrentará a un problema con la composición de movimientos. Cuando se trata de componer dos movimientos uniformes, resultantes de la aplicación simultánea en $B$ de dos impulsos instantáneos (fig. 9), uno según $B C$, el otro según $B A$, basta aplicar la ley del paralelogramo, y la composición de fuerzas se corresponde con la composición de movimientos. Si los tramos $B C, B A$ y $B D$ se recorren en el intervalo de tiempo $\Delta t$, entonces la cantidad de fuerza motriz de cada una de las componentes y de la resultante es proporcional al movimiento que ha generado en dicho $\Delta t$, es decir, será como cada uno de estos tramos. Supongamos ahora que la fuerza dirigida según $B A$ no es un impulso instantáneo, sino una fuerza de intensidad constante que actúa a lo largo de todo el $E t$. En este caso, Galileo demostró que la trayectoria entre $B$ y $D$ es un arco de parábola. La cantidad motriz de esta fuerza será como su intensidad compuesta con el intervalo $\Delta t$.

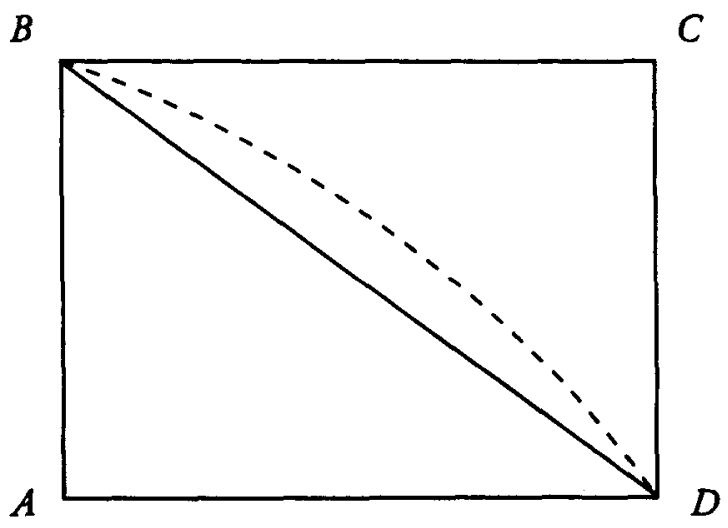

Fig. 9 
En el caso del movimiento circular, la intensidad sigue siendo constante, pero la dirección de la fuerza varía en cada instante. El paralelogramo se deforma, y la ley sólo será aplicable si estipulamos que la medida de la cantidad motriz es la de esta misma fuerza aplicada en línea recta, o si admitimos que cometemos un error muy pequeño o nulo suponiéndola constante durante un intervalo infinitesimal de tiempo. A esta última suposición se le ha denominado "aproximación parabólican, pues equivale a aproximar el arco de circunferencia $\longrightarrow$ de cualquier trayectoria, si la intensidad variable de la fuerza también se supone constantepor un arco de parábola. Lo que permite aplicar en este tramo la ley de Galileo.

La historiografía tradicional supone que Newton aprendió de Hooke, en 1679, la idea de una composición del movimiento inercial por la tangente con una fuerza centrípeta, y no ya centrífuga. La discusión de esta cuestión se aleja demasiado de nuestros objetivos, de modo que dejaremos de lado esta correspondencia, salvo por una breve alusión más adelante. La historia subsiguiente es bien conocida ${ }^{84}$. Dicho a grandes rasgos, Edmond Halley visitó a Newton en 1684, preguntándole por la ley del movimiento planetario. Los términos exactos de la pregunta han sido objeto de debate entre los historiadores; pudo tratarse de cuál sería la trayectoria de un planeta bajo una ley inversamente proporcional al cuadrado de la distancia, o bien, como I.B. Cohen considera más probable, la índole de esta ley dada una órbita elíptica ${ }^{85}$. Newton le respondió que había estudiado este problema cinco años atrás (y la cuestión es si lo hizo antes o después de la correspondencia con Hooke), pero no pudo encontrar los papeles - según dijo- por lo que reconstruyó la demostración (volveremos más adelante sobre este punto en concreto). El resultado fue el pequeño tratado de Motu, del que existen varias versiones con ligeras variantes 86. En 1685 Newton realizó una importante revisión y ampliación, depositando el nuevo manuscrito en la Biblioteca de la Universidad en calidad de texto de una serie de clases impartidas en 1684-5, conocido como las Lectures de Motu, si bien, dado que su editor Whiteside lo califica así, aludiremos a él

84 En NEwTON $(1726 ; 1987)$, la introducción de E. Rada resume esta evolución y sus problemas, suministrando abundante bibliografia. Un estudio mucho más detenido es el de COHEN (1971).

85 COHEN (1971), págs. 50-51.

86 Existen cinco versiones. Las tres que se conservan en la Biblioteca de la Univ. de Cambridge se recogen en HERIVEL (1965), quien las numera I, II y III, correspondientes a sus Mss. IXa, IXb, IXc, de acuerdo con una reconstrucción cronológica. Vtanse las págs. 93-94 y 257. Hall y Hall (1978), págs. 237-292, presentan la versión III y la comparan con otras dos anteriores. 
como a la revisión inicial del de Motu. A este manuscrito le seguiría el conjunto de borradores que condujo a la primera edición de los Principia ${ }^{87}$.

En el de Motu, Newton se ocupó de nuevo del movimiento circular. Comparó dos movimientos circulares, estableciendo que sus fuerzas - ahora claramente expresadas como centrípetas- son como los cuadrados de los arcos descritos en el mismo tiempo, divididos por los radios; un resultado equivalente al anterior. Ahora (fig. 10a) la desviación $D C$ ya no se dirige al centro, sino que sigue la dirección de una cuerda $D F$, determinada por la condición de que el tramo inercial $B C$ tenga la misma longitud que el arco $B D .^{88}$ De modo que Newton adopta aquí la cuerda de la evoluta discutida anteriormente, y representada aquí por el pequeño arco $D C$ que, como las líneas de trazos $G F$ y $G S$, se han anadido a la figura original. Pero todavía, en la revisión inicial del de Motu realizada inmediata o casi inmeditamente después (fig. 10b), toma la desviación $C D$ en $D$ paralela al radio $B S^{89}$. Las tres direcciones de la desviación empleadas sucesivamente por Newton, todas ellas convergentes hacia $B S$ cuando $D$ se aproxima a $B$, se muestran en la figura 11 .

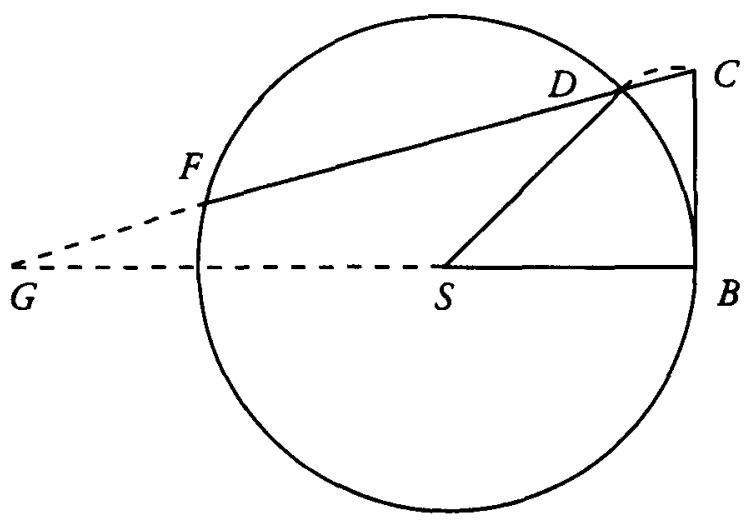

Fig. 10a

87 El manuscrito, bajo el título "The revised treatise De Motu Corporum», se transcribe integramente en WHITESIDE (1967-81), Vol. VI, págs. 92-228. Para un seguimiento detenido de los manuscritos, véanse las introducciones de Whiteside en dicho volumen, y el estudio de COHEN (1971).

88 HeRIVEL (1965), págs. 259-260, y WhITESIDE (1967-81), Vol. VI, págs. 36 y 38.

89 WHITESIDE (1967-81), Vol. VI, págs. 128 y 130. 


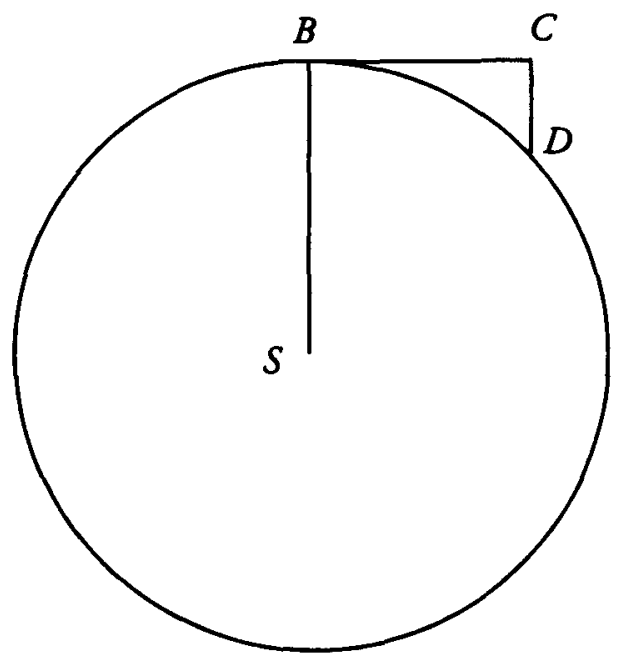

Fig. 106

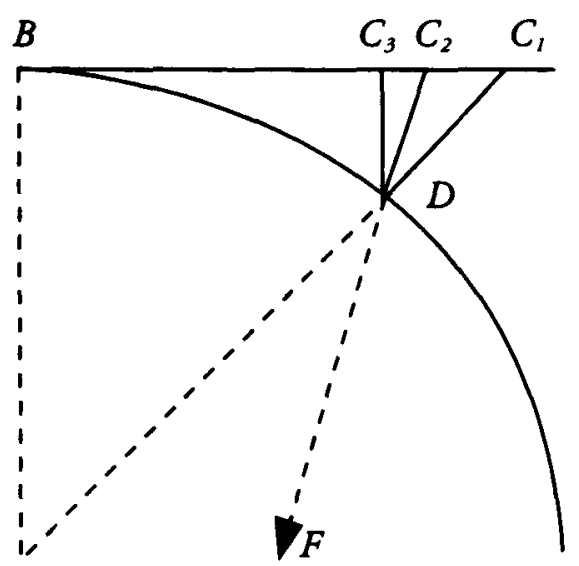

Fig. 11

En el caso de la desviación centrífuga $D C_{1}$, como hemos visto, Newton no está componiendo dos movimientos, uno inercial uniforme y otro radial acelerado. Pero tal composición de movimientos sí entra en juego en su análisis del movimiento circular bajo una fuerza centripeta. En este caso la desviación es la $C_{2} D$, que resulta de igualar las longitudes del arco $B D$ y del tramo inercial $B C_{z}$ Newton pudo haber seguido el complicado razonamiento de 
Huygens, pero quizás se pudo limitar a razonar así: Dado que la fuerza centrípeta siempre es perpendicular a la dirección del movimiento inercial, no contribuirá en nada a éste, siendo su único efecto el de curvar la trayectoria. Por consiguiente, dado que $B D$ y $B C_{2}$ se recorren en el mismo intervalo de tiempo, su longitud deberá ser la misma. Si Newton vio que con ello aproximaba un pequeño arco de evoluta, no lo mencionó.

Resta la cuestión de las razones por las que finalmente adoptó la desviación $C_{3} D$. Esta aparece por primera vez en el de Motu, aplicada al movimiento en una elipse (salvando el caso de un manuscrito que se analizará más adelante, que pudiera datar de 1679$)^{90}$. Y se aplica al movimiento circular en la primera revisión de este manuscrito ${ }^{91}$. De modo que es hora de ver lo que pasa cuando la fuerza centrípeta no sólo cambia continuamente de dirección, sino también de intensidad. Lo que nos devuelve a la aproximación poligonal.

Del infinitesimal al indivisible

Entre los papeles de Newton se encuentra un manuscrito titulado «A Demonstration that the Planets by their gravity towards the sun may move in Ellipses". De éste se encuentra también una copia entre los papeles de Locke, que lleva la fecha de marzo de 1689/90. Sin embargo, se considera posible que fuese compuesto antes de los Principia, más probablemente en 1679 o 1684.

La ley de Kepler se enuncia como la Prop. 1:

«Si un cuerpo se mueve en el vacío y es atraido continuamente hacia un centro inmóvil, se moverá constantemente en uno y el mismo plano, y en ese plano describirá áreas iguales en tiempos iguales ${ }^{92}{ }$.

90 Ibid., pág. 40.

91 Ibid, pág. 128.

92 «If a body move in vacuo and be continually attracted toward[s] an immoveable center, it shall constantly move in one and the same plane, and in that plane describe equal areas in equall times." HERIVEL (1965), VIII, pág. 246. El manuscrito también se encuentra en HaLI y HaLl (1978), págs. 293-301. 
Para la demostración, Newton recurre al modelo poligonal. En la figura 12, $A$ es el centro atractivo, y se supone que la atracción actúa, no continuamente, sino por impresiones discontinuas en $B, C, D, \ldots$ a intervalos de tiempo que considera como "momentos físicos». Aquí cabe interpretar que estos momentos, a diferencia de los matemáticos (que podrían ser infinitesimales o incluso indivisibles) son cantidades pequeñas, pero finitas. Como en el caso del movimiento circular, $C I$ es la trayectoria inercial que hubiese seguido el cuerpo de no actuar el impulso centrípeto en $B$, que le obliga a seguir según $C D$. Así, el punto $D$ se determina como la intersección de la paralela a $C p$ trazada por $I$ y la paralela a $I C$ trazada por $p$. Como las bases $B C, C I$ de los triángulos $A B C$, $A C I$ son iguales, los triángulos serán iguales (pues su altura, determinada por la perpendicular desde $A$ a la prolongación de la línea $I C B$, es la misma). Asimismo, los triángulos $A C I$ y $A C D$ son iguales. Pues ambos tienen la misma base $A C$, y siendo $D I$ paralela a $A C$, su altura será la misma. Y por consiguiente el triángulo $A C D$ será igual al $A B C$, cumpliéndose esto para los triángulos $A D E, A E F$, etc., delimitados por las sucesivas impulsiones en $E, F$, etc. Así, las áreas compuestas de estos triángulos iguales serán entre sí como los tiempos invertidos en describirlas. $Y$, finalmente:

"Supóngase ahora que los momentos de tiempo disminuyen en longitud $y$ aumentan su número in infinitum, de modo que los impulsos o

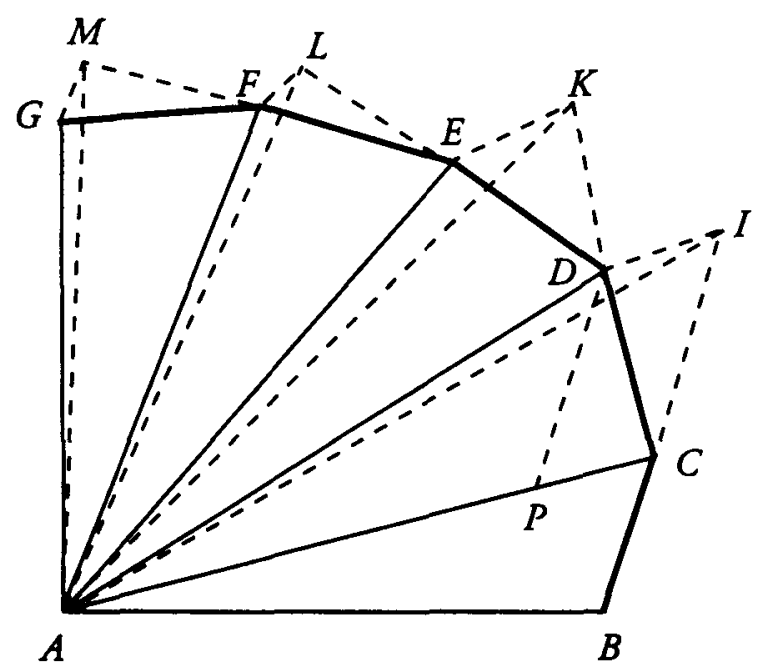

Fig. 12 
impresiones de la atracción pueden tornarse continuos y que la línea $\mathrm{BCDEFG}$, por el número infinito y la infinita pequefiez de sus lados $\mathrm{BC}$, $\mathrm{CD}, \mathrm{DE}$, etc., puede convertirse en una curva: y el cuerpo por la atracción continua describirá áreas de esta curva $\mathrm{ABE}, \mathrm{AEG}, \mathrm{ABG}$, etc., proporcionales a los tiempos en que son descritas" ${ }^{93}$.

Esta demostración se repite, sin cambios significativos aparentes, en las distintas versiones del de Motu y en las ediciones de los Principia. Sin embargo, de acuerdo con nuestra interpretación, antes del abandono del infinitesimal por parte de Newton la "curva» resultante no es la rigurosa, sino la línea poligonal infinitangular que se considera equivalente a ella; pero después, reduciéndose los intervalos de tiempo entre impactos, no a infinitesimales de tiempo, sino a indivisibles, la multiplicación al infinito (ahora actual) del número de impactos dará lugar a la curva rigurosa.

En la primera versión del de Motu todavía sigue con la curva poligonal:

"Háganse ahora estos triángulos infinitamente pequeños e infinitos en número, tales que a cadauno de los momentos de tiempo corresponda un solo triángulo, actuando ahora la fuerza centrípeta sin interrupción, y la proposición quedará demostradan ${ }^{94}$.

Se ve que todavia aquí los "momentos» de tiempo son infinitesimales, en la medida en que, al aumentar infinitamente el número de impactos, los intervalos entre ellos aún delimitan triángulos. Pero en la casi inmediata revisión inicial del de Motu, en donde se incorpora el método de las razones primeras y últimas, las cosas cambian ${ }^{95}$. El texto es idéntico al de la cita anterior, pero finaliza de forma distinta:

93 «Suppose now that the moments of time be diminished in length and encreased in number in infinitum, so that the impulses or impressions of the attraction may become continuall and that the line BCDEFG by the infinite number and infinie littleness of its sides BC, $\mathrm{CD}, \mathrm{DE}$ etc. may become a curve one: and the body by the continual attraction shall describe areas of this Curve $A B E, A E G, A B G$ etc. proportionall to the times in which they are described." Ibid., pág. 248.

94 "Sunto jam haec triangula numero infinita et infinitè parva, sic, ut singulis temporis momentis singula respondeat triangula, agente vi centripeta sine intermissione, et constabit propositio.» WHITESIDE (1967-81), Vol. VI, pág. 34.

95 Whiteside data el primer tratado de Motu en el otoho de 1684, y la primera revisión en el invierno o a principios de la primavera de 1684/85. 
«(...) sin interrupción, de modo que el área SAF [corresponde a la ABG en nuestra figura] sea la suma última de los triángulos evanescentes, y entonces, por el Corolario 4 del Lema III, la proposición quedará demostrada” ${ }^{96}$.

Dada una curva acotada por dos sucesiones de rectángulos, incritos y circunscritos, el Lema III establece que, cuando dichas bases disminuyen al infinito, la razón última entre ambas sucesiones es la de igualdad. El Corolario 4, que estas figuras últimas - las que se obtienen finalmente cuando, al disminuir las bases de los rectángulos en ambas sucesiones, éstas llegan a coincidirno son rectilíneas, sino los limites curvilineos de figuras rectilineas ${ }^{97}$. El Lema III y el Corolario indicado se expresan del mismo modo en los Principia, en donde la demostración de la ley de Kepler finaliza en términos similares a la de la revisión del de Motu.

"Auméntese ahora el número de triángulos y disminúyase su altura in infinitum y su perimetro último $A D F$ [ADG en nuestra figura] será una línea curva (por el Corolario 4 del Lema III); y por tanto la fuerza centripeta, por la que un cuerpo es continuamente separado de la tangente de dicha curva, actúa continuamente; (...)" ${ }^{98}$.

Retornemos ahora a los manuscritos denominados «Kepler-motion papers" donde, como se ha visto, y si aceptamos la fecha de composición de 1679, aparece por vez primera la demostración, vía el modelo poligonal, de la ley de Kepler. Según la lectura de Erlichson, en las dos proposiciones restantes Newton también aplica el modelo del impacto ${ }^{99}$. Sin embargo, sigue presentando las deducciones de Newton en términos de límites, lo que en nuestra interpretación no sería admisible hasta la primera revisión del de Motu; lo cual supone una toma de postura respecto de la fecha de redacción, que asumiremos definitivamente como anterior a la del de Motu. Veamos las proposiciones.

96 "(...) sine intermissione, utque area SAF sit summa ultima triangulor evanescentium, et per Corollarium quartum Lemmatis tertij constabit propositio." WHITESIDE (1967-81), Vol. VI, pág. 124.

97 Ibid., págs. 108 y 110.

98 Augeautur jam numerus $8 \mathrm{C}$ minuatur latitudo triangulorum in infinitum; \& corum ultima perimeter ADF, (per corollarium quartum lemmatis tertii) erit linea curva: ideoque vis centripeta, qua corpus a tangente hujus curvae perpetuo retrahitur, aget indesinenter; (...).» NEWTON (1726; 1972), pág. 90, y trad. de E. Rada, pág. 174.

99 ERLICHSON (1991a). 
La Prop. II establece que, para un cuerpo moviéndose en una elipse bajo la acción de una fuerza central dirigida hacia uno de sus focos, la atracción en los extremos $A$ y $C$ de la elipse (fig. 13) será recíprocamente como los cuadrados de sus distancias al foco en dichos puntos. Newton lo prueba gracias a la ley de las áreas, suponiendo que los arcos $A E, C D$ se describen en tiempos iguales, por lo que las áreas $A F E, D F C$ serán iguales. Suponiendo que los arcos $A E$, $C D$ «son tan pequeños que pueden tomarse por líneas rectas» ${ }^{100}$, los rectángulos $1 / 2 A F \cdot A E$ y ${ }^{1 / 2} F C \cdot D C$ resultan iguales, y $A E: C D:: F C: F A$. Si se trazan los segmentos $E M$ y $D N$ perpendiculares, respectivamente, a las tangentes $A M$ y $C N$ en $A$ y $C$, dada la igual curvatura de la elipse en estos puntos tales segmentos serán entre sí como los cuadrados de los arcos $A E \mathrm{y} C D$, y por lo tanto $E M: D N:: F C^{2}: F A^{2}$. En el mismo tiempo en que recorre estos arcos, en ausencia de la fuerza centrípeta hubiese recorrido los tramos $A M$ y $C N$, así que las atracciones son como las distancias $E M$ y $D N$, y por tanto las atracciones en $A$ y $C$ serán como $F C^{2}$ a $F A^{2}$.

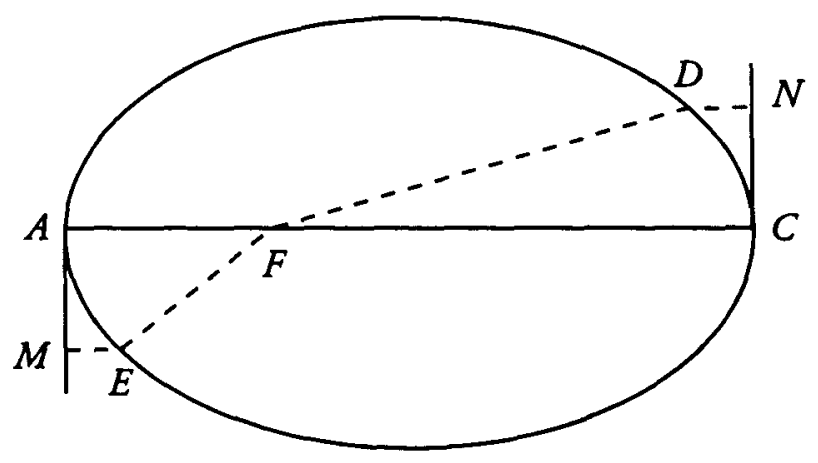

Fig. 13

Según Erlichson, como hemos dicho, Newton también se basa en el modelo poligonal en esta demostración. Las líneas poligonales que considera son las cuerdas $A E$ y $C D$. En la figura 12 se ve que, como las fuerzas motrices en los vértices del polígono son proporcionales a los cambios de velocidad que generan, la

100 "To be so very short that they may be taken for right linesw, HERIVEL (1965), VII, pág. 248. 
razón de las fuerzas motrices en dos puntos como $E$ y $C$, será como la razón entre las líneas $L F$ e $I D$. Del mismo modo, en la figura 13 la razón entre las fuerzas motrices en $A$ y $C$ será como la razón en el límite entre $E M$ y $D N$. Y añade:

"La aproximación en la Prop. 2 es la misma que en la Proposición 1, basada en un modelo poligonal de intervalos de tiempo iguales y en el paso al límite cuando $\Delta t \rightarrow 0$. La evidencia del polígono de intervalos de tiempo iguales, suprimido, es el hecho de que las áreas AFE y CDF en la Figura son iguales. Para Newton, no valía la pena mostrar el resto de la elipse dividida en áreas iguales, puesto que las únicas áreas iguales de interés en esta proposición eran las de los dos extremos de la elipse” ${ }^{101}$.

Alternativamente, podríamos decir que Newton, que toma $A E$ y $C D \tan$ cortos que se pueden sustituir por líneas rectas, los está considerando infinitesimales y sustituyendo el arco por la cuerda. ¿Es esto el modelo poligonal? Si lo es, entonces toda aplicación del cálculo infínitesimal no es más que la aplicación de este modelo, y Erlichson puede encontrarlo doquiera que aquél se emplee. Todavía hay que admitir que Newton, en esta proposición, no dice explícitamente que los arcos considerados sean infinitesimales.

Otro aspecto curioso es la dirección de la desviación, perpendicular a la tangente. Dentro de la suposición de Erlichson, se explica inmediatamente por la aplicación del paralelogramo de composición de movimientos.

Veamos ahora la Prop. III. En ella Newton demuestra que si un cuerpo describe una elipse, siendo atraído a uno de sus focos, la atracción será recíprocamente como el cuadrado de la distancia del cuerpo al foco. Supone:

"(...) al tiempo dividido en partes iguales y que aquellas partes son muy pequefias, de modo que puedan considerarse como momentos físicos, y que la atracción actúa, no continuamente, sino por intervalos al principio de cada momento físico (...) $n^{102}$.

101 ERLICHSON (1991a), pág. 847.

102 aLet us suppose the time to be divided into equal parts and that those parts are very little ones so that they may be considered as physical moments and that the attraction acts not continually but by intervalls once in the beginning of every physical moment (...).» HERTVEL (1965), VIII, pág. 252. 
En la figura 14, sean $P$ e $Y$ los puntos en que se producen dos de estos impulsos, y $P X$ la distancia sobre la tangente que hubiese recorrido el cuerpo en ese "momento físico" de no actuar la fuerza centrípeta. La línea $X Y$ está generada por la fuerza de atracción y debe ser proporcional a ella y paralela a $P F^{103}$. Análogamente sucede en el punto $p$ de la órbita. Tras alguna manipulación geométrica, se llega a que la atracción en $P$ es a la atracción en $p$ como $X Y$ es a $x y$, y éstas son como $(P Q / X I) \cdot p F^{2}$ a $(p q / x i) \cdot P F^{2}$. El último paso de la demostración es el siguiente:

"Supóngase ahora que los tiempos iguales en los que el cuerpo que orbita describe las líneas $P Y$ y $p y$ se tornan infinitamente pequenos, por lo que la atracción puede tornarse continua y el cuerpo por esta atracción orbita en el perímetro de la Elipse: y las líneas $P Q, X I$ y también $p q, x i$, al volverse coincidentes $y$ en consecuencia iguales, las cantidades $(P Q / X I) \cdot p F^{2}$ y $(p q / x i) \cdot P F^{2}$ se tornarán $p F^{2}$ y $P F_{n}{ }^{104}$.

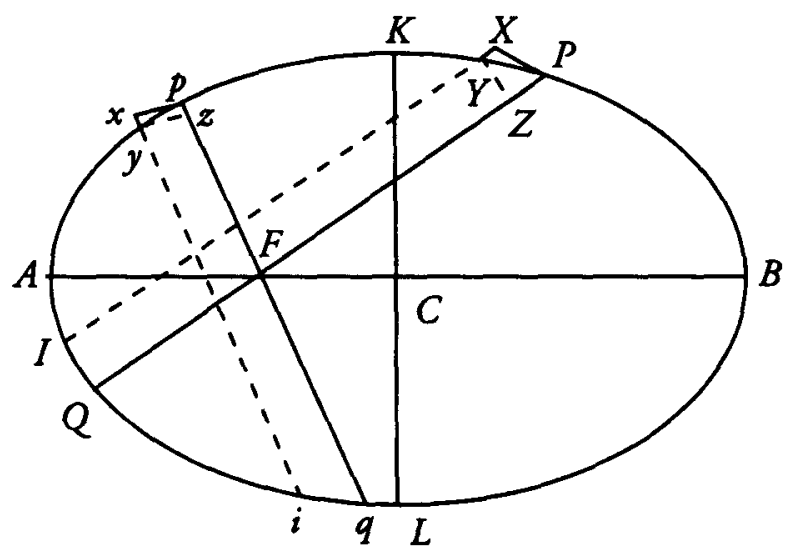

Fig. 14

103 Aqui Herivel observa que este paralelismo, que Newton asume sin prueba, se sigue de la suposición de que $X$ e $Y$ son los puntos, sin y con fuerza centrípeta, a los que llegaría el cuerpo simultáneamente. Pero para ello -afiade- serla necesario reemplazar la elipse por un polígono inscrito. Ibid., pág. 255, nota 12.

${ }_{104}$ "Suppose now that the equal times in which the revolving body describes the lines $P Y$ and $p y$ become infinitely little, so that the attraction may become continual and the body by this attraction revolve in the perimeter of the Ellipsis: and the lines $P Q, X I$ as also $p q, x i$ becoming coincident and by consequence equal, the quantities $(P Q / X I) \cdot p P^{\text {mand }}$ and $(p q / x i) \cdot P P^{2 m a n d}$

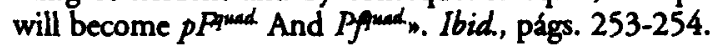


Nótese que dice que cuando los tiempos se tornan infinitamente pequeños -y así las líneas se tornan infinitesimales-, la fuerza se torna continua y el cuerpo se mueve sobre la elipse. Pero obsérvese que, en rigor, las líneas $P Q, X I$ y las $p q, x \dot{i}$ no coincidem, sino que se encuentran infinitamente próximas. Por ello, Herivel observa que el proceso al límite de Newton es en realidad un proceso doble. Pues, en primer lugar, la elipse ha sido reeemplazada por un polígono inscrito; y, en segundo, porque los resultados ya obtenidos son sólo aproximados; cuando la curva no se «aproxima» por un polígono inscrito, sólo se necesita el segundo tipo de proceso al límite ${ }^{105}$. La cuestión se aclara si se considera que el "primer" paso al límite no conduce sino al infinitesimal —en la reducción de los intervalos de tiempo interviene un infinito potencial-, mientras que el segundo conduce a un punto -interviniendo el infinito actual y reduciéndose estrictamente a cero los intervalos de tiempo-. Ahora bien, lo que dice Newton es que, si $P Y$ y $p y$ se tornan infinitamente pequenos, las líneas $P Q, X I$ y las $p q, x i$ coinciden. Parece que esto sólo se puede compatibilizar si aqú, para Newton, la elipse es una línea poligonal de infinitos lados; del mismo modo que, en su primer estudio del movimiento circular por el modelo de impacto, el círculo es un polígono de infinitos lados. Y esto concuerda perfectamente con la conclusión de la Prop. 1 que se citó anteriormente: la línea poligonal, "por el número infinito y la infinita pequefiez de sus lados (...) puede convertirse en una curva."

Se entiende así que Westfall opinase que Newton oscurece el problema comenzando con un concepto de fuerza basado en el modelo de impacto para terminar con uno basado en el modelo de caída libre, y la correspondiente crítica de Erlichson observando que Newton comienza con una fuerza motriz y termina con una fuerza motriz ${ }^{106}$. La razón entre las intensidades de la fuerza en dos puntos de la elipse se obtiene gracias a la razón de las cantidades de fuerza motriz medidas en el mismo intervalo de tiempo.

Sea cual fuere el momento en que se escribió el manuscrito, de lo dicho anteriormente se ve que el marco conceptual que preside estas demostraciones es anterior a la primera redacción del de Motu. Éste muestra la transición entre las dos etapas del cálculo de Newton. En el Teorema I, la deducción de la ley de las áreas de Kepler, citada más arriba, todavía se identifica la curva rigurosa con la línea poligonal de lados infinitesimales. Cuando, en el Teorema 2,

106 WESTFAll (1971), pág. 431, y ERLICHSON (1991a), pág. 847. 
compara la fuerza centrípeta en dos movimientos circulares, parte de la curva rigurosa, como se vio al final del apdo. anterior. Pero en el Teorema 3 Newton calcula la intensidad de la fuerza centrípeta en un punto de una trayectoria curva generah aunque en la figura aparezca una elipse. La demostración anterior ha sido sustituida.

En la figura 15, Newton supone que el cuerpo describe la línea $A P Q$ bajo la acción de una fuerza dirigida hacia $S$. Y, sin explicar sus motivos, toma la desviación $Q R$ paralela a $S P$.

"Digo que la fuerza centripeta será inversamente como la razón $\left(\mathrm{SP}^{2} \cdot \mathrm{QT} \mathrm{T}^{2}\right) / \mathrm{QR}$, supuesto que la cantidad de esta razón se tome siempre como aquella en que se convierte al final cuando los puntos $P$ y $Q$ coinciden" ${ }^{107}$.

En la figura «indefinidamente pequeña" [indefinite parva] $Q R P T, Q R$ varia, en un tiempo dado, como la fuerza centrípeta y, cuando la fuerza es dada, como el cuadrado del tiempo. Cuando ni el tiempo ni la fuerza están dados, variará como ambos. Como el tiempo es el que se invierte en recorrer $P Q$, entonces éste es proporcional al área $S P Q$, o al doble de la misma $S P^{2} \cdot Q T^{2}$. Así,

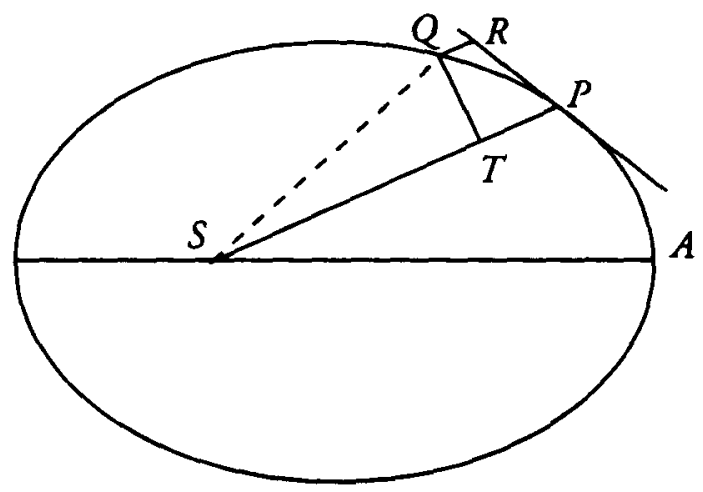

Fig. 15

107 ('...) dico quod vis centripeta sit reciproce ut solidum (SPquad! $Q^{\text {quad. }}$ )/QR, si modo solidi illius ea semper sumatur quantitas quae ultimo fit ubi coeunt puncta $P$ et $Q$.» HeRrveI (1965), DX(1), pág. 260, y WhITESIDE (1967-81), Vol. VI, pág. 40. 


$$
Q R \propto F_{c}(\Delta t)^{2} \propto\left(S P^{2} \cdot Q T^{2}\right)
$$

de donde:

$$
F_{c} \propto \frac{1}{\frac{S P^{2} \cdot Q T^{2}}{Q R}}
$$

En los «Kepler-motion papers», Newton obtiene la fuerza centrípeta en una trayectoria elíptica aplicando el modelo de impacto y comparando las cantidades motrices de las fuerzas centrípetas en dos puntos distintos de la trayectoria. En el de Motu, parte de la curva rigurosa para obtener asimismo la fuerza centrípeta del movimiento circular en dos circunferencias distintas. En el primer caso, las desviaciones se recorren con movimiento uniforme; en el segundo, uniformemente acelerado.

En el primer caso, Newton comparó las cantidades motrices de dos impulsos instantáneos. Dado que identifica la curva rigurosa con la «aproximación" poligonal infinitangular, la fuerza centrípeta "continua" está constituida por una sucesión de impulsos separados por el mismo intervalo infinitesimal de tiempo. Suponiendo dos puntos $A$ y $B$, se podría escribir:

$$
\frac{\Delta(m v)_{A}}{\Delta(m v)_{B}}=\frac{I_{d A}}{I_{d B}}
$$

Supongamos ahora la curva rigurosa, y que cada uno de estos impulsos instantáneos imprime la misma cantidad motriz que la fuerza centrípeta rigurosa de intensidad $I_{i}$

$$
\frac{\Delta(m v)_{A}}{\Delta(m v)_{B}}=\frac{I_{d A}}{I_{d B}}=\frac{I_{e A} d t}{I_{e B} d t}=\frac{I_{e A}}{I_{e B}}
$$

Se ve que, siendo iguales los intervalos de tiempo, la razón entre las intensidades -infinitesimales- de los impulsos instantáneos es la misma que la razón entre las intensidades de la fuerza continua. En este caso de obtención de la fuerza por comparación de las cantidades motrices en dos puntos, pues, tanto da que las desviaciones se recorran con velocidad constante o aceleración 
constante. En el primer caso los espacios son como las velocidades y los tiempos, y así, dado el tiempo, como las intensidades instantáneas; en el segundo, son como las intensidades continuas y los cuadrados de los tiempos, y dado el tiempo, como dichas intensidades. Por consiguiente, tanto si se usa la línea poligonal como la curva rigurosa, las fuerzas serán como las desviaciones.

Naturalmente, si se quiere hallar la fuerza en un punto, ésta ya no será como la desviación, pues intervendrá el intervalo de tiempo, y los resultados son distintos en el caso de que la desviación se recorra con movimiento uniforme o con movimiento uniformemente acelerado. En el caso de la curva rigurosa también es esencial determinar la dirección de la desviación, pues -dado el intervalo de tiempo- de ella depende el espacio recorrido.

En el Teorema 3 del de Motu la desviación en $Q$ se toma paralela a la dirección de al fuerza en $P$. Esta es la desviación que se adopta en el modelo poligonal, fruto de la aplicación del paralelogramo de composición de movimientos. La cantidad de la fuerza motriz en el tramo entre $P$ y $Q$, sin embargo, no se ha aproximado por un único impulso en $P$, pues Newton establece que la desviación $R Q$ se recorre con movimiento uniformemente acelerado. La aproximación que aquí efectúa, a primera vista, es la que se ha denominado "parabólica", suponer que en el tramo entre $P$ y $Q$ la fuerza es constante en magnitud y dirección: el arco $P Q$ se ha sustituido por un arco de parábola. Sin embargo, al aplicar la ley de las áreas, Newton supone que dicho arco se aproxima por la cuerda, al aproximar el área $S P Q$ por el área del triángulo, $1 / 2 S P^{2} \cdot Q T^{2}$. Esto nos introduce en los trabajos de Whiteside, Cohen y Aiton.

La falacia del segundo orden

La crítica de Whiteside y Aiton, que Erlichson ha denominado "la falacia del segundo orden", consiste en defender que la demostración de la ley de las áreas en la Prop. I sólo es válida para porciones infinitesimales de arco. En la demostración del Teorema 3 del de Motu, de hecho -como por otra parte siempre que la usa Newton en los Principia- se hallaría correctamente aplicada, dado que el arco PQ es infinitesimal. Esto supone que en realidad Newton no habría aproximado el arco por la cuerda, sino por una línea poligonal de infinitos lados cuyas longitudes son infinitesimales de segundo orden. 
En el razonamiento de Aiton se supone que (fig. 16) el cuerpo recorre un arco infinitesimal $A B$ bajo la acción de una fuerza centrípeta en un tiempo $d t$. Este intervalo de tiempo se divide en $n$ partes, y la fuerza continua se sustituye por $n$ impulsos instantáneos que actúan al comienzo de cada uno de estos intervalos. Si el cuerpo tiene masa unidad, cada uno de estos impulsos crea una velocidad $f(d t / n)$, siendo $f$ la [intensidad de la] fuerza centrípeta. La distancia atravesada en el primer intervalo de tiempo es $f(d t / n)^{2}$, en el segundo, dado que se conserva la velocidad creada en el primer impulso, será $2 f(d t / n)^{2}$, etc. De modo que:

$$
T B=(1+2+\ldots+n) f\left(\frac{d t}{n}\right)^{2}=\frac{1}{2} n(n+1) f\left(\frac{d t}{n}\right)^{2}
$$

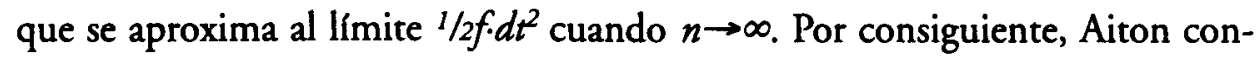
cluye que representar una curva por una serie de arcos parabólicos infinitesimales de primer orden equivale a representar la curva por un polígono cuyos lados son infinitesimales de segundo orden. $Y$ esto, en concreto, se aplica al arco considerado en nuestra figura 12, la demostración de la ley de las áreas ${ }^{108}$.

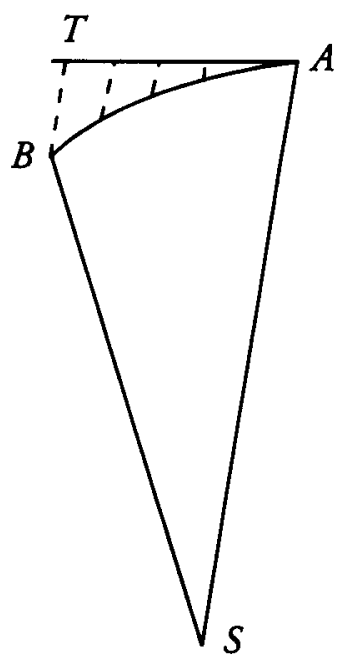

Fig. 16

108 ArTon (1989) págs. 210-211. También se hace esta crítica en ArTon (1972). 
La crítica de Whiteside se desarrolla con referencia a la figura $17^{109}$. En la fig. 17(a), la desviación, en el intervalo de tiempo entre $B$ y $C$, es el segmento infinitesimal $C c$, y en el tiempo total de $B$ a $F$ viene medida, en longitud y dirección, por $\gamma \delta$, paralela a $B S$. Análogamente, la desviación entre $C$ y $D$ es $D d$ y en el intervalo $C F$ viene medida por $\delta \varepsilon$. Así, la desviación en la totalidad de impulsos entre $B$ y $E$ está representada por el arco poligonal $\gamma \delta \varepsilon \ldots F$, que en el límite se convierte en el arco $\gamma q F$, precisamente la evoluta (fig. 17b). Para cualquier punto $p$ sobre el arco $B F$, se cumple que el área del triángulo Spq es igual a la del sector PSF.

Para que se puedan despreciar estas «sutilezas» — como las llama Whiteside y supone que hace Newton- es preciso que la fuerza se considere constante a lo largo del arco $B F$. Y para ello este arco debe ser infinitesimal. De este modo, si en $B F$ se producen $n$ impactos y se recorre en $d t$, cada desviación $C c=D d=E e=\ldots=f(d t / n)^{2}$. La desviación total en el arco $B F$ será el arco poligonal $\gamma \delta \varepsilon \ldots F$, y como:

$$
\gamma \delta=n f\left(\frac{d t}{n}\right)^{2} ; \delta \varepsilon=(n-1) f\left(\frac{d t}{n}\right)^{2} ; \ldots
$$

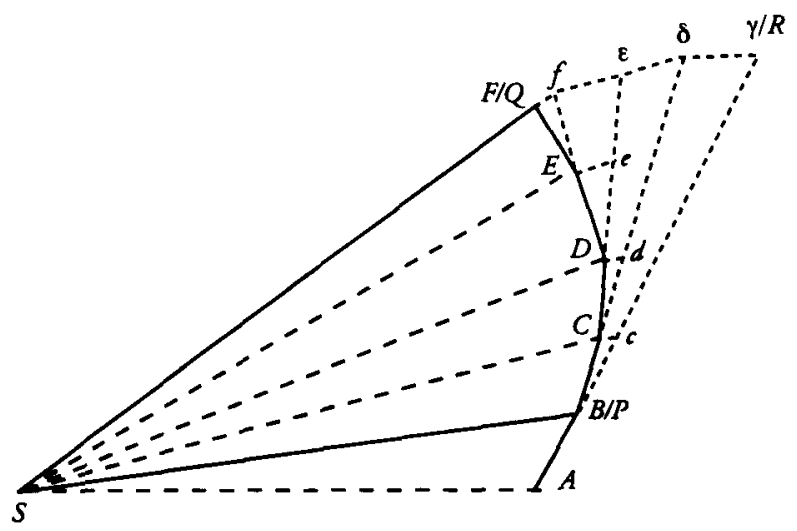

Fig. $17 a$

109 WhITESIDE (1967-81), Vol. VI, págs. 35-37, nota 19. La crítica apareció por primera vez en WHTTESIDE (1966), recensión de HERVEL (1965). La repitió de nuevo en WHITESIDE (1970), y en esta edición de los trabajos matemáticos de Newton de 1989. 
la longitud de la desviación total será:

$$
\gamma F=\sum_{i=0}^{n-1}(n-i) f\left(\frac{d t}{n}\right)^{2}=\frac{1}{2}\left(1+\frac{1}{n}\right) f d t^{2}
$$

precisamente el valor de la ec. (22) que obtiene Aiton. Dicho arco tiene una pendiente máxima en $F$, precisamente la de $S F$, y se odrá aproximar en magnitud y dirección por cualquier línea entre $F$ y $B$ cuya pendiente no exceda la de $S F$.

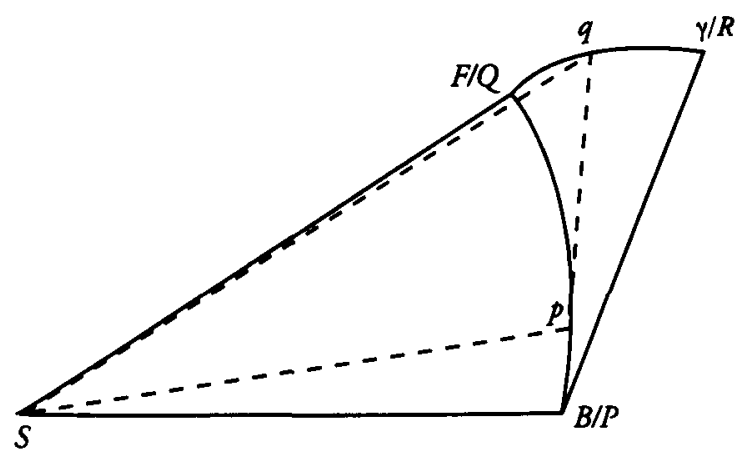

Fig. 176

Tal como ha señalado Erlichson, ${ }^{110}$ el problema con estas interpretaciones es que, por un lado, parten de una concepción moderna de la fuerza centrípeta y, por otro, quizá debido a esto mismo, mezclan el modelo de impacto con la aproximación parabólica. Como se ha subrayado antes, la "fuerza total", o la cantidad motriz de la fuerza centripeta, en el arco $B F$, es la suma escalar de las cantidades motrices de los impactos en él producidos, siendo cada uno de ellos proporcional al desplazamiento $C c, D d, .$. , dado que dichos desplazamientos se producen todos en el mismo intervalo de tiempo, el que se da entre impactos sucesivos. Pero estas desviaciones, fruto de los impulsos en los vértices, no se

110 ERLICHSON (1992b). 
recorren (como suponen Whiteside y Aiton al hacerlas proporcionales al cuadrado del tiempo) con movimiento uniformemente acelerado, sino con movimiento inercial.

Supongamos, pues, que el tramo $B F$ se recorre en un tiempo infinitesimal. Cada desviación se recorre con movimiento uniforme, luego $C c=D d=\ldots=f_{M}$ $(d t / n)$, en donde $f_{M}$ es la cantidad motriz del impulso instantáneo impreso al comienzo de cada intervalo de tiempo. La desviación total en el tramo $B F$, calculada como la suma de las desviaciones individuales, será:

$$
n f_{M}\left(\frac{d t}{n}\right)=f_{M} d t
$$

resultado independiente del número, finito o infinito, de impactos. Calculada según Whiteside, la longitud de esta nueva desviación total serfa:

$$
\gamma F=\sum_{i=0}^{n-1}(n-i) f_{M}\left(\frac{d t}{n}\right)=\frac{1}{2}(n-1) f_{M} d t
$$

que, comparada con (25), da:

$$
f_{M}=f\left(\frac{d t}{n}\right)
$$

de modo que $f_{M}$ tiene con $f$ la misma relación que $I_{d}$ con $I_{e}$ en nuestra ecuación (7). A diferencia de $f, f_{M}$ es función del número $n$ de impactos en que se divide el intervalo. Cuando aquél tiende a infinito, es infinitesimal; en el límite, se anula estrictamente.

Whiteside dirige tres reproches a Newton en relación con esta demostración:

1) Ignora si se ha introducido algún error significativo en la acción total al suponer que la fuerza continua (que varía en dirección a lo largo del arco infinitesimal entre impactos) se halla adecuadamente representada por un impulso instantáneo equivalente aplicado en un punto. 
2) No justifica su suposición de que el «arco-límite poligonal» se convierte en un único arco orbital cuando el número de impulsos se convierte en infinito.

3) Supone inmediatamente que la dirección y longitud de esta desviación está unívocamente —e intuitivamente, añade- determinada.

En términos, generales, la crítica de Whiteside abarca todos los modelos de impacto empleados por Newton, incluido el tratamiento del movimiento circular en el "Waste Book». Dado que el pensamiento de Newton respecto del análisis se desarrolla en cuanto menos dos etapas, conviene darles respuesta después de que, ya con todos los elementos pertinentes de sus manuscritos mecánicos en la mano, se recapitule toda la exposición anterior.

A esta interpretación de Whiteside se le puede asociar el estudio de Cohen acerca de las dos definiciones de fuerza en los Principia. Cohen parte de la idea -que bemos asumido aquil - de que para Newton la entidad fundamental no es la intensidad de la fuerza, sino su cantidad motriz. Pero limita esta cantidad motriz a intervalos de tiempo infinitesimales, en la forma de impulsos cuasiinstantáneos:

«En la presentación que sigue, entenderé por impulso una «fuerza» que actúa durante un tiempo muy breve, como cuando una bola de acero al final de un péndulo golpea a otra; o cuando una raqueta de tenis golpea una pelota. Me referiré a tales "fuerzas" o impulsos como instantáneos, con el fin de contrastarlos can las "fuerzas" continuas como la gravedad y otras "fuerzas centrípetas" (ipero naturalmente no son absolutamente instantáneas en el sentido de no requerir ningún tiempo para su acción!)» ${ }^{111}$.

Desde esta concepción, inevitablemente Cohen alude al papel del tiempo como regulador del cambio, y casi llega a sugerir para el tiempo una estructura granular:

«Una consecuencia de un flujo de tiempo universal o uniforme es que el incremento de tiempo es constante bajo todas las circunstancias. Esto es equivalente a un concepto cuasi-discreto de los infinitesimales de un tiempo variable que no fluye del todo continuamentes ${ }^{112}$.

111 COHEN (1970), pág. 144. Las itálicas son suyas.

112 Ibid., pág. 179. 
De hecho, Newton acarició esta idea en una etapa muy temprana, durante sus primeros años de estudiante en Cambridge; pero halló que la idea de unos átomos de tiempo entendidos como entidades conceptualmente indivisibles no se podía sostener. Otra alternativa, más próxima a los Principia, es considerar - como hizo Barrow- que el continuo está compuesto de infinitesimales; serian, aunque divisibles, todos iguales por naturaleza ${ }^{113}$. $Y$ esto es lo que quiere significar Cohen: el tiempo es continuo a nivel finito y sólo a nivel infinitesimal es discreto ${ }^{114}$. Pero adviértase que esta concepción nos devuelve -en cierta medida - a la idea de que las trayectorias descritas por los cuerpos son líneas poligonales de lados correspondientemente infinitesimales. Digo en cierta medida, porque la idea de Cohen - y de Whiteside y Aiton- es más sutil. Pues en cada uno de estos tramos infinitesimales la curva rigurosa se habría aproximado por un arco de parábola el cual, a su vez, estaría aproximado por una línea poligonal infinitangular cuyos lados serian infinitesimales de segundo orden. Lo primero supone que, en un intervalo de tiempo infinitesimal, la fuerza se considera constante en magnitud y dirección; lo segundo, que dicho intervalo se compone a su vez de infinitos tramos infinitesimales en los que la velocidad se considera constante. Lo que equivale a decir que una fuerza constante en un intervalo de tiempo infinitesimal de primer orden está compuesta de una sucesión de impulsos instantáneos impresos sobre el cuerpo a intervalos de tiempo que son infinitesimales de segundo orden ${ }^{115}$.

En una primera etapa, en la que Newton identifica la curva rigurosa con la línea poligonal, estas concepciones no son aplicables. Pues la aproximación parabólica constituye un elemento que se encuentra entre la línea poligonal y la "verdadera" curva. Serían en todo caso aplicables cuando, partiendo de la curva rigurosa, Newton aproxime la fuerza en un arco de la misma considerándola constante, o cuando llegue a ella desde una aproximación poligonal mediante un paso al límite. Ahora bien, cuando se parte de la aproximación poligonal y se aumenta al infinito el número de impactos, se llega a la curva rigurosa sin pasar por la aproximación parabólica. Un arco de parábola es una curva, y por consiguiente puede ser el límite de una sucesión de líneas poligonales, pero no pertenecer a ella. Así, desde este modelo se puede responder inmediatamente a los reproches de Whiteside:

113 Sobre estas cuestiones véase SELLEs (1999), en prensa.

114 COHEN (1970), pág. 185.

115 Ibid., pág. 181. 
1) La fuerza continua (que varía en dirección a lo largo del arco infinitesimal entre dos impactos) no se halla adecuadamente representada por un impulso instantáneo equivalente porque las intensidades de ambos son inconmensurables. Cabe pensar que, probablemente, Newton no ignoraba esto, pues es algo que se ve inmediatamente al plantearse, en la aproximación poligonal, el cálculo de la intensidad en un punto (a diferencia, como vimos, de la razón entre las intensidades en dos puntos).

2) Newton no necesita justíficar lo obvio. La sucesión de curvas poligonales tiende a un único arco orbital porque tiende a un único límite, lo que tiene que ver con que,

3) la ley de composición de movimientos, dado el movimiento inicial y el intervalo de tiempo, determina unívocamente el primer vértice de la línea poligonal, y a partir de éste, sucesivamente, todos los demás, cualquiera que sea el intervalo de tiempo entre ellos.

Resta por ver el caso en que se parte de la curva rigurosa. En éste un arco de la misma se aproxima por un arco de parábola si en él la fuerza se supone constante en magnitud y dirección. Se podria suponer que tal es el caso del Teorema 3 del de Motu, convertido en la Prop. VI de los Principia. En este caso, las consideraciones anteriores tampoco son pertinentes. Porque, para que lo fuesen, Newton tendría que haber aplicado, bajo la aproximación anterior, el paralelogramo de composición de movimientos entre los puntos $P$ y $Q$, en cuyo caso tendría que atender a todas las sutilezas que expone Whiteside. Como se ha argumentado anteriormente, dicho paralelogramo sólo se puede establecer rigurosamente en el límite, con lo que en realidad, si ha de seguir considerándose un paralelogramo, sólo puede concebirse como virtual. Pero ni más ni menos virtual que el caso de la velocidad instantánea que tratamos en el primer apartado de este trabajo Como all anticipamos, la teorla de infinitesimales y la de límites constituyen métodos complementarios y excluyentes... a menos que situemos los nuevos infinitesimales "dentro" de los viejos puntos.

Conclusión: El desarrollo de la mecánica y las etapas del cálculo

En una primera etapa, Newton identifica la línea poligonal infinitangular con la curva rigurosa Esto está claro en el tratamiento del movimiento circular mediante impactos que se recoge en el "Waste Book". El circulo es un polígono 
de infinitos lados. Siendo así, este polígono "último» no constituye ningun tipo de aproximación, y en esta concepción la fuerza, centrifuga o centrípeta, se concibe continua cuando se halla constituida por una sucesión de impulsos infinitesimales separados por intervalos infinitesimales de tiempo iguales. Aquil la expresión para la fuerza continua de nuestra ecuación (6) no es aplicable. Sí Newton llega aquí a un resultado correcto es gracias a dos razones. Una es que compara dos cantidades de fuerza motriz en el mismo intervalo de tiempo. En la figura 5, la desviación $y c=b d$ se recorre en el mismo tiempo en que se recorre ab. El empleo del mismo intervalo de tiempo está relacionado con la medida de la fuerza y con la aplicación del paralelogramo de composición de movimientos. La otra razón es que el perímetro del "último" de los polígonos, aunque no es la circunferencia del círculo, difiere de ella tan sólo en una cantidad infinitesimal.

Según la interpretación tradicional, que en este punto también comparte Erlichson, el impulso instantáneo en cada vértice constituiría una conceptualización de la actuación continua de la fuerza sobre el arco comprendido entre dicho vértice y el siguiente. Si se admite la identificación entre la línea poligonal y la curva rigurosa, entonces tal conceptualización no es posible.

Resulta, sin embargo, muy probable que Newton escribiese esta demostración después de sus consideraciones sobre el movimiento circular a partir del caso de una bola rodando por el interior de un cilindro. En esta última entrada aparece la comparación entre los impactos elásticos de dos cuerpos deformables y dos cuerpos duros, así como la consideración de que se puede concebir que una línea curva consiste en una infinitud de tramos rectilíneos, o bien en cualquier punto de la curva el movimiento se puede concebir según la tangente. Esta consideración supone que en este momento Newton distingue entre la línea poligonal y la curva rigurosa como concepciones alternativas, pero no presenta indicios de que considere que la primera supone tan sólo una aproximación a la segunda

Su siguiente cálculo de la fuerza centrifuga del movimiento circular parte de la curva rigurosa y considera que la desviación se recorre con movimiento uniformemente acelerado. En este caso, la intensidad de la fuerza es constante, pero no asi su dirección. Según la interpretación tradicional, la suposición de Newton es que en un tiempo muy pequeño se puede considerar que la dirección de la fuerza es constante, "a la manera de la gravedad». Nuestra interpretación es diferente. Siendo la fuerza centrifuga un conatus, no imprime ningún 
desplazamiento real, y en un desplazamiento virtual no hay razón alguna para suponer que se aproxima una fuerza de intensidad variable por otra de intensidad constante. Por consiguiente, no se puede hablar en este caso de una «aproximación parabólica».

La siguiente fuente de interés para nosotros es la construcción de la órbita que efectúa Newton en su correspondencia con Hooke de 1679. Aquí considera el movimiento de un cuerpo en el interior de la Tierra en un corte diametral, bajo la acción de una fuerza gravitatoria constante. Sin entrar en más detalles, diremos que el cuerpo parte de $A$ (fig. 18) con un movimiento inercial según $A M$. De acuerdo con Newton,

«(...) el movimiento del cuerpo en $G$ está compuesto del movimiento que tenía en $\mathrm{A}$ hacia $\mathrm{M}$ y de todos los innumerables movimientos convergentes sucesivamente generados por las impresiones de la gravedad en cada momento de su tránsito desde A hasta G: (...)" ${ }^{116}$.

E inmediatamente después alude a:

«Los movimientos innumerables e infinitamente pequeños (pues considero aquí el movimiento de acuerdo al método de indivisibles) continuamente generados por la gravedad en su tránsito de A a F $(. ..){ }^{117}$.

Movimientos que son proporcionales al tiempo en que son generados.

Erlichson ha reconstruido los razonamientos de Newton en términos del modelo de impacto, dentro del contexto del debate sobre la aportación de Hooke al análisis del movimiento centrípeto ${ }^{118}$. Para ello parte de impulsos espaciados a iguales intervalos de tiempo $\Delta t$ y llega a la trayectoria en el límite, cuando $\Delta t \rightarrow 0$. Ciertamente, Newton pudo aplicar aquí el modelo de impacto del mismo modo que lo hizo en el "Waste Book" en el caso del movimiento

116 "For the motion of the body at $G$ is compounded of the motion it had at $A$ towards $\mathrm{M}$ and of all the innumerable converging motions successively generated by the impresses of gravity in every moment of its passage from A to G: (...)." HERIVEL (1965), VII, pág. 244.

117 "The innumerable and infinitly little motions (for I here consider motion according to the method of indivisibles) continually generated by gravity in its passage from $\mathrm{A}$ to $\mathrm{F}$ (...).» Ibid.

118 ERLICHSON (1992a). Sobre esta cuestión existe toda una polémica en curso, en la que no entraremos. 


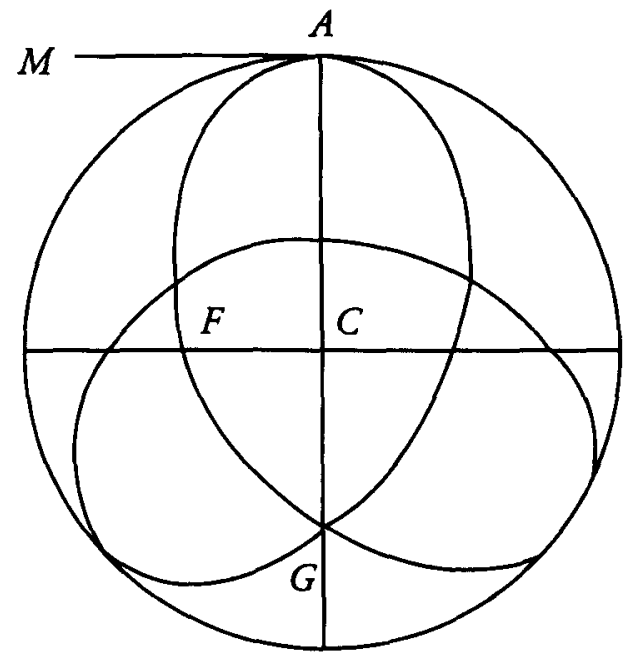

Fig. 18

circular. Y el hecho de que al final de la carta escriba que podría afiadir algo sobre la descripción de la curva "por puntos quam proxime" ${ }^{119}$ sugiere que pudo emplear este método para aproximar la forma de la curva. De todos modos, lo que Newton sí especifica es que emplea el «método de los indivisibles», denominación que entonces aludía al cálculo mediante infinitesimales.

Parece, pues, razonable suponer que en una segunda etapa, previa al método de las primeras y últimas razones, Newton trabaja con entidades infinitesimales (se supone que dentro del marco conceptual del método fluxional). Cuando, conocida la fuerza, se trata de determinar la trayectoria, se puede partir, dada una velocidad inicial, del modelo de impacto, llegando a una línea poligonal de lados infinitesimales, que considera equivalente a la curva rigurosa. Cuando, conocida la trayectoria, quiere obtener la fuerza, aplica el cálculo infinitesimal y aproxima la curva rigurosa $\longrightarrow$ el tramo o tramos pertinentes de la misma - por la línea poligonal que la inscribe, tomando así la cuerda por su arco.

En nuestra reconstrucción, los «Kepler-motion papers" serían contemporáneos o inmediatamente posteriores a esta carta a Hooke, de acuerdo con la

119 Herrvel (1965), VII, pág. 244. 
opinión de Westfall. En ellos demuestra la Prop. 1, la ley de las áreas, mediante el modelo del impacto, deteniéndose como hemos visto en la línea poligonal de lados infinitesimales en la que la atracción se torna "continua". En la Prop. 2, cuando calcula la fuerza de atracción en los dos extremos de la elipse, Erlichson interpreta que se sigue aplicando el modelo del impacto, vía su demostración anterior de la ley de las áreas. Pero también pudo haber aplicado el cálculo infinitesimal. Newton no habla de impulsos y no dice que las desviaciones se recorran con movimiento uniforme o acelerado. A estas alturas debía saber que, tanto considerase una fuerza compuesta de impulsos o actuando continuamente, las intensidades buscadas serán como las desviaciones dado que ha escogido dos puntos en los extremos de la elipse, que tiene la misma curvatura. El caso equivale al de comparar dos movimientos circulares Pero en la Prop. III busca comparar la fuerza en dos puntos cualesquiera de la elipse, y lo anterior no sirve. Por ello parte del modelo de impacto, que ahora menciona explícitamente (cuando en la Prop. 2 no lo hace) y busca la fuerza en el límite en que los tiempos se hacen infinitamente pequeños (pero no nulos), de modo que llega a la línea poligonal. $Y$ - según nuestra reconstrucción - algo falla: los puntos $P$ y X, $p$ y $x$, no coinciden- pese a lo que afirma - sino que se quedan a una distancia infinitamente próxima.

Si fue éste el manuscrito que prometió buscar a Halley, y si lo encontró, quizás entonces al revisarlo Newton debió darse cuenta del problema. Sea como fuere, el hecho es que la demostración desapareció en el de Motu, siendo sustituida por la determinación de la fuerza centrípeta en un punto cualquiera de una cónica. En lo que se refiere a la mecánica, aquí asoma por primera vez el método de las razones. Pues - véase la figura 16- Newton establece que la relación válida sólo será "aquélla en que se convierte en el límite cuando los puntos $P$ y $Q$ coinciden».

Visto desde el método de las razones, el motivo por el que Newton toma la desviación $R Q$ paralela a la dirección $S P$ de la fuerza en $P$ está clara: no es porque sustituya la fuerza en el tramo $P Q$ por un único impulso en $P$, o porque la considere constante, sino porque $R Q$ es la dirección de la fuerza centripeta en el punto $P$. Es cierto que esta dirección se corresponde con la que hace constante la fuerza, en magnitud y dirección, en el tramo $P Q$. Pero Newton debió hacer en realidad una suposición distinta, aunque a la postre equivalente: que la desviación $R Q$ se recorre con una fuerza constante en magnitud y dirección porque este es el caso en el punto $P$; en un punto, la fuerza no varía. La figura «indefinidamente pequena” (indefinidamente pequeria, no infinitesimal) $Q R P T$ sólo 
constituye una aproximación, tanto mejor cuanto más se aproxime $Q$ a $P$. Incluso en el caso de que el arco $P Q$ fuese infinitesimal, todavía sería una aproximación. Sólo se alcanzará la exactitud cuando, en el límite, $Q$ coincida con $P$. En tal límite, el área $S P^{2} . Q T^{2}$ se habrá desvanecido en una línea, y la desviación $Q R$, en un punto. Pero, para Newton, todavía existirá la razón. Desde su teoría de límites y su concepción del infinitesimal en geometría, la «aproximación parabólica» es sólo precisamente eso: una aproximación, un paso intermedio del proceso que conducirá, llevado estrictamente a su término, al valor exacto que se buscaba.

Llegado a este punto, Newton revisa completamente el de Motu, y comienza a transformarlo en un verdadero tratado. En una primera revisión anade un Lema muy significativo, que demuestra por el método de las razones:

"La distancia que un cuerpo describe desde el principio de su movimiento bajo la acción de una fuerza centrípeta cualquiera está en la razón duplicada del tiempo» ${ }^{120}$.

En los Principia se convertirá en el Lema X, explicitándose que se aplica a fuerzas constantes o continuamente crecientes o decrecientes. Así se justifica la «aproximación parabólica» mediante la aplicación de la ley de Galileo entendida dinámicamente.

En este conjunto de revisiones del de Motu se inscriben dos borradores con definiciones, el primero de los cuales presenta un elemento muy significativo respecto del giro que el problema de la determinación de la fuerza centrípeta en un punto obligó a dar a Newton. En el primero de ellos, como la Def. 16, se expresa la nueva concepción de "momentos" que se citó en nuestro primer apdo. Los momentos son "principios de generación o alteración". Principios, y no las mismas generaciones o alteraciones, que son cantidades finitas. Entre tales principios, que son "como un punto respecto de una línea", se cuenta la fuerza centrípeta "o cualquier otra fuerza momentánea" como momento del impetus. Esta definición de "momento" no aparece en posteriores versiones del manuscrito; pero el concepto se explicitará en el Lema II del Libro II de los Principia.

120 "Spatium quod corpus urgente quacunque vi centripeta ipso motus initio describit esse in duplicata ratione temporis." HERIVEL (1965), IX, pág. 295. 
En nuestro apdo. relativo a la aproximación poligonal y a la curva rigurosa analizábamos lo que sucede cuando, en el límite, una fuerza continua se resolvía en un impulso instantáneo o una sucesión de ellos. En tanto en cuanto Newton trabajase con una línea poligonal de infinitos lados, sólo podía obtener la razón entre las cantidades de fuerza motriz en dos puntos distintos, pero al hallarse ausente la idea de que ésta era una aproximación a la curva rigurosa, curva que se alcanzaba mediante un estricto paso al límite, no había justificación para suponer que dicha razón igualaba a la razón entre las intensidades. El mismo concepto de intensidad sólo cobra sentido en la curva rigurosa. Vimos que, cuando un impacto elástico con deformación se conceptualiza mediante un impacto instantáneo, esto sólo se puede hacer igualando sus cantidades motrices, pues si se efectúa un paso al límite la intensidad del impulso instantáneo asociado se torna indeterminada. En el caso de que la acción motriz de una fuerza constante se sustituya por una sucesión de impulsos instantáneos decíamos, haciendo referencia a la figura 1, que en el caso de la línea poligonal de lados infinitesimales el rectángulo se había reducido a una suma de rectángulos de base infinitesimal. En el límite, cuando se alcanza la curva rigurosa, dichos rectángulos se han convertido en líneas, y la cantidad motriz de cada impulso se ha "desvanecido". Aquí, las líneas constituyen las intensidades, en cada punto, de la fuerza continua inicial. Pero se ve que, si se establece la razón entre las cantidades de dos impulsos instantáneos antes de alcanzar el límite, y se hace decrecer el intervalo de tiempo hasta anularse, las cantidades motrices habrán desaparecido, pero quedará la razón entre estas cantidades "últimas», límites de las cantidades que se desvanecen -en este caso el límite geométrico del rectángulo es su lado, una línea- y la razón entre dichas líneas será la razón entre las intensidades de la fuerza continua.

Precisamente por ello, para obtener la intensidad de la fuerza continua en un solo punto habrá que expresarla en términos de una razón entre cantidades partiendo de la curva rigurosa: la de la desviación al cuadrado del tiempo invertido en recorrerla. En este sentido, el Lema X del Libro 1 de los Principia, que establece la proporcionalidad entre los espacios y los cuadrados de los tiempos en el verdadero comienzo del movimiento es crucial. La dirección de la desviación, mucho menos. Porque en otro lema crucial, el Lema XI, Newton despeja de un plumazo las sutilezas de Whiteside al establecer en el caso 2 que, en el momento de evanescencia, atendiendo a su inclinación, todas las desviaciones son equivalentes. 


\section{Bibliografia}

Arton, E. J. (1972): The Vortex Theory of Planetary Motions, Londres: Macdonald y Nueva York: Elswevier.

Arron, E. J. (1989): «Polygons and Parabolas: Some Problems Concerning the Dynamics of Planetary Orbits", Centaurus, 31, 207-221.

Brackenrudge, J. B. (1995): Ihe Key to Newton's Dynamics. The Kepler Problem and the Principia, Berkeley, Los Angeles, Londres: Univ. of California Press.

GLAGETT, M. (1968): "Some Novel Trends in the Science of the Fourteenth Century", en Art, Science, and History in the Renaissance, ed. Ch. Singleton, Baltimore, Maryland. The Johns Hopkins Press, págs. 275-303. Reimpreso en M Clagett, Studies in Medieval Physics and Mathematics, Londres: Variorum Reprints, 1979.

CoHen, I. B. (1970): «Newton's Second Law and the Concept of Force in the Principias, en The Annus Mirabilis of Sir Isaac Newton, 1666-1966, eid. R. Palter, Cambridge (Mass.) Y Londres:The M.I.T. Press, págs. 143-185. El comentario de J. E. McGuire, en págs. 186-191.

CoHen, I. B. (1971): Introduction to Newton's Principia, Cambridge: Univ. Press.

COSTABEL, P. (1983): La signification d'un débat sur trente ans (1728-1758). La question des forces vives, Paris: Centre National de la Recherche Scientifique.

DE GANDT, F. (1987): L'oeuvre de Torricelli: Science galikéenne et nouvelle géométrie, Publications de la Faculté des Lettres et Sciences Humaines de Nice, n. $32\left(1 .^{\mathrm{a}}\right.$ ser.), París: Les Belles Lettres.

De GandT, F. (1995): Force and Geometry in Newton's Principia, Princeton (N.J.): Princeton Univ. Press.

ERLISCHSON, H. (1991a): «Motive force and centripetal force in Newton's mechanics», Am.J.Phys., 59 (9), 842-849.

ERLISCHSON, H. (1991b): «How Newton Went from a Mathematical Model to a Physical Model for the Problem of a First Power Resistive Force», Centaurus, 34, 272-283.

ERLISCHSON, H. (1992a): "Newton and Hooke on Centripetal Force Motion", Centaurus, 35, 46-63.

ERlischson, H. (1992b): "Newton's Polygon Model and the Second Order Fallacy", Centaurus, 35, 243-258.

Hall, A. R., y Hall, M. B. (Eds.), (1962): Unpublished Scientific Papers of Isaac Newton. A selection from the Portsmouth Collection in the University Library, Cambridge, Cambridge: Univ. Press. 
Hankins, T. L. (1970): Jean d'Alembert. Science and the Enlightenment, Oxford: Clarendon Press.

Herrvel, J. (1965): The Background to Newton's Principia. A Study of Newton's Dynamical Researches in the Years 1664-84, Oxford: Clarendon Press.

MCGuire, J. E., y TAMnY, M. (1983): Certain Philosophical Questions. Newton's Trinity Notebook, Cambridge: Univ. Press.

NeWTON, I. (1726; 1972): Isaac Newton's Philosophiae naturalis principia mathematica, 3.a ed. (1726): con anotaciones de A. Koyré, I. B. Cohen y A. Whitman, Cambridge (Ingl.), Univ. Press, y Cambridge (Mass.): Harvard Univ. Press, 2 Vols.

Newton, I. (1726; 1987): Principios matemáticos de la filosofia naturah ed. de E. Rada. Madrid: Alianza Editorial, 2 Vols.

NEWTON, I. (1930; 1977): Optica, o tratado de las reflexiones. refracciones, inflexiones y colores de la luz, 4. ${ }^{a}$ ed. inglesa (1730). Ed. de C. Solís. Madrid: Alfaguara.

ScOTT, W. L. (1970): The conflict between atomism and conservation theory, 16441860, Nueva York: Science History Publications.

SELLÉs, M. A. (1996): "Prólogo", en J. Simón, La génesis de la mecánica de fluidos (1640-1780), Madrid: UNED, págs. XI-XXV.

Selles, M. A. (1999): "Isaac Newton y el infinitesimal», Theoria, en prensa.

SHEA, W. R. (1993): La magia de los números y el movimiento. La carrera cientifica de Descartes, Madrid: Alianza Editorial. (Ed. orig. Watson Publishing International, 1991).

Solts, C. (1987): «La fuerza de Dios y el éter de Cristo: La explicación de la interacción a través del espacio en la filosofia de la naturaleza de Newton", Sylva Clius, 1 (n. $\left.{ }^{\circ} 2\right), 51-80$.

WestFall, R. S. (1971): Force in Newton's physics. The science of Dynamics in the Seventeenth Century, Nueva York: Science History Publications.

WeSTFALl, R. S. (1980): Never at Rest. A Biography of Isaac Newton, Cambridge: Univ Press.

Whiteside, D. T. (Ed.) (1967-81): The Mathematical Papers of Isaac Newton, Cambridge: Univ. Press, 8 Vols. 